\title{
The retinoic acid receptor co-factor NRIP1 is uniquely upregulated and represents a therapeutic target in acute myeloid leukemia with chromosome $3 q$ rearrangements
}

\author{
Sarah Grasedieck, Ariene Cabantog, ${ }^{2}$ Liam MacPhee, ${ }^{2}$ Junbum Im,${ }^{2}$ Christoph Ruess, ${ }^{3}$ Burcu \\ Demir, ${ }^{3}$ Nadine Sperb, ${ }^{3}$ Frank G. Rücker, ${ }^{3}$ Konstanze Döhner, ${ }^{3}$ Tobias Herold, ${ }^{4}$ Jonathan R. \\ Pollack, ${ }^{5}$ Lars Bullinger, ${ }^{6}$ Arefeh Rouhi ${ }^{2 \#}$ and Florian Kuchenbauer ${ }^{2 \#}$
}

${ }^{1}$ University of British Columbia, Department of Microbiology \& Immunology, Vancouver, British Columbia, Canada; ${ }^{2}$ Terry Fox Laboratory, BC Cancer Agency, Vancouver, British Columbia, Canada; ${ }^{3} \mathrm{Ulm}$ University Hospital, Department of Internal Medicine III, Ulm, Germany; ${ }^{4}$ Department of Medicine III, University Hospital, LMU Munich, Munich, Germany; ${ }^{5}$ Department of Pathology, Stanford University School of Medicine, Stanford, CA, USA and ${ }^{6}$ Department of Hematology, Oncology and Tumor Immunology, Charité University Medicine, Berlin, Germany.

\author{
Correspondence: \\ Florian Kuchenbauer \\ fkuchenbauer@bccrc.ca \\ Received: $\quad$ November 16,2020 \\ Accepted: $\quad$ November 25, 2021 \\ Prepublished: December 2, 2021. \\ https://doi.org/10.3324/haematol.2020.276048 \\ (2022 Ferrata Storti Foundation \\ Haematologica material is published under \\ a CC-BY-NC license @ (1) @
}

${ }^{\#} A R$ and FK contributed equally as co-senior authors.

\begin{abstract}
Aberrant expression of Ecotropic Viral Integration Site 1 (EVI1) is a hallmark of acute myeloid leukemia (AML) with inv(3) or $\mathrm{t}(3 ; 3)$, which is a disease subtype with especially poor outcome. In studying transcriptomes from AML patients with chromosome 3q rearrangements, we identified a significant upregulation of the Nuclear Receptor Interacting Protein 1 (NRIP1) as well as its adjacent non-coding RNA LOC101927745. Utilizing transcriptomic and epigenomic data from over 900 primary samples from patients as well as genetic and transcriptional engineering approaches, we have identified several mechanisms that can lead to upregulation of NRIP1 in AML. We hypothesize that the LOC101927745 transcription start site harbors a context-dependent enhancer that is bound by EVI1, causing upregulation of NRIP1 in AML with chromosome 3 abnormalities. Furthermore, we showed that NRIP1 knockdown negatively affects the proliferation and survival of 3qrearranged AML cells and increases their sensitivity to all-trans retinoic acid, suggesting that NRIP1 is relevant for the pathogenesis of inv(3)/t(3;3) AML and could serve as a novel therapeutic target in myeloid malignancies with $3 q$ abnormalities.
\end{abstract}

\section{Introduction}

Ectopic activation of the Ecotropic Viral Integration Site 1 (EVI1) gene is associated with a dismal outcome in patients with acute myeloid leukemia (AML) or myelodysplastic syndrome (MDS), who have an average survival of only 10 months after diagnosis. ${ }^{1}$ EVI1 is located in the MDS1 And EVI1 Complex Locus (MECOM) on chromosomal band 3q26.2, encodes a DNA-binding protein with two zinc finger domains, and is expressed in hematopoietic stem and progenitor cells. In an inducible mouse model, Evi1 overexpression led to the suppression of erythropoiesis and lymphopoiesis, driving a pre-leukemic expansion of myeloid cells which ultimately led to leukemic transformation, ${ }^{2}$ suggesting that activation of EVI1 drives myeloid leukemias. Moreover, ectopic activation of the $E V / 1$ gene through vector integration was associated with the development of AML in a gene therapy trial. ${ }^{3}$ Although upregulated EVI1 is the defining molecular char- acteristic of AML with inv(3) or $\mathrm{t}(3 ; 3)$, high EVI1 expression has been reported in approximately $11 \%$ of all adult AML cases, in which it was suggested to be an independent adverse prognostic factor., ${ }^{1,-7}$ Currently, there are no targeted therapies or additional prognostic indicators available for myeloid malignancies with abnormal $3 q$ or high EVI1 expression and the mechanisms that cause or contribute to EVI1 upregulation remain largely unclear.

In 2012, Haferlach et al. reported seven AML cases with translocation $\mathrm{t}(3 ; 21)$ (q26;q11). This led to the formation of an EVI1 fusion protein with the Nuclear Receptor Interacting Protein 1 (NRIP1), which the authors found to be associated with an especially poor prognosis. ${ }^{8}$ Additionally, a more recent report described a poor prognosis, therapy-induced childhood AML with a cryptic t(3;21)(q26;q11), leading to NRIP1-EVI1 fusion, which displayed high EVI1 expression. ${ }^{9}$ While studying transcriptomes from AML and MDS patients with or without $3 q$ rearrangements, we discovered that both NRIP1 as well 
as its neighboring non-coding gene, LOC101927745, were upregulated in $M E C O M$-rearranged $A M L$ without $t(3 ; 21)$ fusions, such as inv(3) and $\mathrm{t}(3 ; 3)$.

NRIP1 and its neighboring gene LOC101927745, share a topologically associating domain (TAD). TAD are genomic regions that are delimited and insulated from external regulatory influences by CCCTC-binding factor (CTCF)- and cohesinbound sites. ${ }^{10}$ Genes and regulatory elements within a TAD were shown to physically interact with each other more frequently than with sequences located outside their TAD, ${ }^{10}$ prompting us to investigate whether a potential co-regulatory relationship exists between NRIP1 and LOC101927745 which could lead to upregulation of NRIP1 in MECOM-rearranged AML. Although NRIP1 has been implicated in differentiation processes via modulation of retinoic acid (RA) receptors ${ }^{11,12,13}$ and regulation of energy metabolism, ${ }^{14}$ its role in myeloid malignancies is largely unknown. Hypothesizing that NRIP1 could function as a yet undescribed proto-oncogene in AML cells, we utilized public transcriptomic and epigenomic data collected from more than $900 \mathrm{AML}$ and MDS patients and conducted genetic and transcriptional engineering approaches to: (i) identify potential mechanisms that can lead to upregulation of NRIP1 and (ii) investigate how the perturbation of NRIP1 expression would affect inv(3)/t(3;3) AML cells.

\section{Methods}

\section{Patients' data and experimental datasets}

Most datasets that were analyzed for this study are available either through the National Cancer Institute's Genomic Data Commons portal (TCGA-LAML, ${ }^{15}$ Beat AML, ${ }^{16}$ https://portal.gdc.cancer.gov/), the International Human Epigenome Consortium data portal (BLUEPRINT primary AML samples, ${ }^{17}$ sample identities: ERS699839, ERS699842, ERS699843, ERS753996; https://epigenomesportal.ca/ihec/) or the National Center for Biotechnology Information's Gene Expression Omnibus database (https://www.ncbi.nlm.nih.gov/gds) under the following identifiers:

GSE114922: healthy and MDS CD34+ cells, RNA-seq $(n=130) ;^{18}$ GSE106291: AMLCG-2008 \& AMLG-1999 AML samples, RNAseq $(n=250) ; 19$

GSE104099: cytogenetically normal AML, AMLSG_07-04, RNA-seq $(\mathrm{n}=46) ;^{20}$

GSE35159: expression profiling in 12 human myeloid cell lines, microarray; ${ }^{21}$

GSE123255: murine leukemic stem cell enriched cells (LSCe) +/- ATRA; 22

GSE31477: ENCODE TF and co-factors in various cell lines, ChIP-seq;23

GSE32465: ENCODE TF and co-factors in various cell lines, ChIP-seq; ${ }^{24}$
GSE36030: murine B10 cell line, Rad21 ChIP-seq (ENCODE mouse project);

GSE136488: murine E14 cell line, Ctcf ChIP-seq (ENCODE mouse project);

GSE55407: THP-1 AML cell line, CTCF and Rad21 ChIP-seq; ${ }^{25}$ GSE87286: SKH-1 AML cell line, ChIP-seq and RNA-seq; ${ }^{26}$ GSE72816: Gm12878 cell line ChIA-PET cluster data; ${ }^{27}$ GSE63525: Gm12878 and K562 Hi-C chromatin contact data;28

PRJNA385337: THP-1 Hi-C chromatin contact data; ${ }^{29}$

GSE52457: H1-derived hMSC Hi-C chromatin contact data; ${ }^{30}$ GSE84662: keratinocytes Hi-C chromatin contact data. ${ }^{31}$

\section{Survival analyses}

Cox proportional hazard and Kaplan-Meier analyses for association with overall or event-free survival were calculated using the $R$ survival ${ }^{32}$ and survminer ${ }^{33}$ packages. Patients were dichotomized into groups expressing the gene of interest at a high or low level based on maximally selected rank statistics. ${ }^{34}$

\section{Patients' samples and cell lines}

RNA sequencing was performed on primary samples of viably frozen bone marrow from patients $(n=65)$ with a complex karyotype (CK-AML) (Online Supplementary Methods). Forty of the 65 (62\%) patients were treated on consecutive multicenter treatment trials of the AML Study Group (AMLSG), applying age-adjusted intensive chemotherapy: AMLHD98A $(n=4$; NCT00146120) and AMLSG07-04 ( $n=22 ;$ NCT00151242) for younger patients (16 to 60 years); AMLSG06-04 ( $n=14$; NCT00151255) for elderly patients (>60 years). All studies were approved by local ethics committees, and all patients gave informed consent to treatment, cryopreservation of samples, and molecular analyses according to the Declaration of Helsinki. All the cell lines used were obtained from the German Collection of Microorganisms and Cell Cultures (DSMZ), except for the Cas9-expressing OCI-AML5 cells which were a gift from Dr. Jan Krönke (Ulm University Hospital, Germany). All cells were maintained in adherence to the culturing conditions recommended by the DSMZ.

\section{All-trans retinoic acid treatment and analysis}

All-trans retinoic acid (ATRA; Sigma-Aldrich, Germany) was prepared in dimethylsulfoxide (DMSO) at $100 \mathrm{mM}$ and further diluted in phosphate-buffered saline. Cell lines that were transfected with NRIP1-targeting GapmeR or control or with NRIP1-targeting shRNA or control after selection (see Online Supplementary Methods) were seeded at $0.25 \times 10^{6}$ cells per well in $2 \mathrm{~mL}$ culture medium at a final concentration of 0.5 $\mu \mathrm{M}$ ATRA or a concentration-matched DMSO control. Cells were analyzed after 24 and $72 \mathrm{~h}$ for cell proliferation by counting trypan-negative cells, for gene expression of NRIP1, MECOM, and LOC101927745, for NRIP1 protein expression (see Online Supplementary Methods), and for apoptosis after 
staining with an annexin-V-APC antibody (Biolegend, USA) and SYTOX-Blue dead stain (Invitrogen, USA) using flow cytometry.

\section{Results}

\section{The NRIP1-EVI1 fusion positions the EVI1 open reading frame under control of the NRIP1 gene regulatory elements} To understand which functional domains of the NRIP1 and EVI1 proteins were lost and retained in a fusion event as reported by Haferlach et al. ${ }^{8}$ and D'Angiò et al., ${ }^{9}$ we studied the exact $\mathrm{t}(3 ; 21)$ (q26;q11) breakpoints and found that the NRIP1EVI1 fusion does not generate a novel chimeric protein, but instead removes most of the upstream regulatory elements of EVI1 and places the complete EVI1 coding sequence (exons 3 to 16) under the control of the three putative NRIP1 promoters and additional upstream regulatory elements (Figure 1A). Similar to what was observed in $\operatorname{inv}(3)(q 21 q 26)$ or $\mathrm{t}(3 ; 3)(\mathrm{q} 21 ; \mathrm{q} 26) \mathrm{AML}$ cases, in which EVI1 was reported to appropriate multiple enhancer sites of the GATA2 gene, ${ }^{35,36}$ we found that at(3;21)(q26;q11) event effectively places the complete $E V I 1$ open reading frame under the control of a different transcriptional network.

\section{Expression of NRIP1 and LOC101927745 is associated with poor survival in acute myeloid leukemia}

Analysis of published RNA-sequencing datasets, comprising sorted healthy human donor bone marrow cell populations $(n=56),{ }^{18}$ blasts from MDS patients $(n=74),{ }^{18}$ and primary AML samples ( $n=950$, including TCGA L-AML, ${ }^{15}$ AMLCG-2008/1999, an in-house generated cytogenetically normal $\mathrm{AML}^{20}$ and a CK-AML cohort $^{37}$ as well as data from the Beat AML trial, ${ }^{16}$ showed that transcript levels of NRIP1, located on chromosome 21q, as well as its neighboring gene, LOC101927745, were highly expressed in $\mathrm{CD} 34^{+}$hematopoietic stem and progenitor cells from healthy donors and MDS patients (Online Supplementary Figure S1A) and were significantly upregulated in inv(3)/t(3;3) AML patients compared to all other cytogenetic AML subgroups ( $P_{\text {adj. }}=0.01$ and 0.003 , respectively) (Figure $1 \mathrm{~B})$. We were able to confirm this exceptionally high expression of LOC101927745 and NRIP1 in EVI/ high - compared to $E V I 7^{\text {low }}$-expressing AML cell lines (total of $\mathrm{n}=4$ vs. $\mathrm{n}=17$ ) (Online Supplementary Figure $1 B-D$ ).

Based on the report by Haferlach et al., which associated the presence of an NRIP1/EVI1 fusion gene with an especially poor prognosis, ${ }^{8}$ we next examined whether NRIP1 and LOC101927745 transcript levels were linked to outcome in AML patients. Indeed, survival analyses using the Beat AML RNA-sequencing dataset ${ }^{16}$ showed that high NRIP1 as well as high LOC101927745 expression were significantly associated with poorer overall survival (Figure 2A), independently of the inclusion of $\operatorname{inv}(3) / t(3 ; 3)$, complex karyotype and also of $\operatorname{del}(5 q) / \operatorname{del}(7 q)$ cases, which represent the most common co- abnormalities of inv(3)/t(3;3) (Online Supplementary Figure S2). The association of high NRIP1 or LOC101927745 expression with poorer outcome was further corroborated in the AMLCG-2008 cohort $^{19}$ as well as in RNA-sequencing data from CK-AML patients ${ }^{37}$ (Figure 2B, C, Online Supplementary Methods). Of note, LOC101927745 and NRIP1 transcript levels exceeded the prognostic stratification capacity of EVI1 levels in all analyzed datasets both when applying a dichotomization approach based on maximally selected rank statistics ${ }^{34}$ (Figure 1A-C, Online Supplementary Figure S2) or the median (data not shown).

In line with other studies reporting EV/1 expression in the absence of detectable EV/1 fusion events or inv(3), only 11/126 (8.7\%) and 5/28 (17.8\%) of EVI1-expressing patients carried molecularly detectable $3 q$ abnormalities in the analyzed Beat AML and CK-AML cohorts, respectively (2x dupl(3q26), $\mathrm{t}(3 ; 21)(\mathrm{q} 26 ; \mathrm{q} 22), \mathrm{t}(3 ; 3)(\mathrm{q} 26 ; \mathrm{q} 21)$, and $\mathrm{t}(3 ; 6)(\mathrm{q} 26 ; \mathrm{p} 22))$, in which expression was defined as EVI1 $>5$ transcripts per kilobase million. Although expression of LOC101927745 and NRIP1 was particularly enriched in inv(3) AML (i.e., EV/ high), and both transcripts showed a high degree of correlation in $E V / 7^{\text {high }}$ healthy and MDS CD34 $4^{+}$blast cells ( $R=0.78$ and 0.89) (Figure 2D), we observed that in AML this correlation was perturbed $(R=0.31$ and 0.64) (Figure 2E). Compared to healthy and MDS samples, in which all NRIP1-expressing cells also expressed LOC101927745, NRIP1 and LOC101927745 RNA transcripts only showed correlation in $46 \%$ of AML cases. However, AML patients who expressed LOC101927745 always expressed NRIP1 (Figure 2E). Considering this highly specific pattern of expression, we next investigated a potential co-regulatory relationship between LOC101927745 and NRIP1 in AML.

\section{The LOC101927745 transcription start site contains an NRIP1 gene regulatory element}

NRIP1 and its neighboring gene LOC101927745 share a TAD (Online Supplementary Figure S3A, B), suggesting a co-regulatory relationship between these genes. In support of this hypothesis, we found that the exonic regions of LOC101927745, its genomic location and its orientation relative to the murine Nrip1 homolog as well as their Ctcf:Rad21 TAD boundaries are conserved between human and mouse genomes (Online Supplementary Figure S3B-D). Of note, the region overlapping the putative transcription start site (TSS) of LOC101927745 is annotated as GH21J015439 in most recent genome browser versions, due to its classification as a candidate enhancer of NRIP1 by the GeneHancer project $^{38}$ (Online Supplementary Figure S4A, based on eQTL and $\mathrm{Hi}-\mathrm{C}$ data). A defining feature of enhancers is that they establish measurable physical contact with the promoters of their regulated genes. As there are currently no chromatin conformation capture data available from cells with chromosome 3 abnormalities, we instead compared $\mathrm{Hi}-\mathrm{C}$ data from NRIP1 low leukemia cell lines (THP-1, K562 and Gm12878) to data generated from human skin samples, which show 
strong NRIP1 as well as EVI1 expression when compared to mature blood cells (Figure $3 \mathrm{~A}$ ). When we subtracted normalized chromatin contact counts obtained in NRIP1 ${ }^{\text {low }}$ AML cell lines from counts recorded in NRIP $7^{\text {high }} / E V I 1^{\text {high }}$ human skin cells, we found that contact was 70- to 330-fold increased specifically between the LOC101927745 TSS (GH21J015439) and the NRIP1 genomic locus in NRIP1/igh -expressing cells
(Figure 3B, blue areas depict regions where contact intensities in NRIP1/EVI ${ }^{\text {high }}$ tissues exceed contact in NRIP1'ow $A M L$ cells). Collectively, these observations suggest that LOC101927745 likely harbors an NRIP1-controlling regulatory element.

To further explore the transcription factor (TF) binding and chromatin state at the LOC101927745/NRIP1 locus in AML cells

A

EVI1 gene locus:

NRIP1 gene locus:
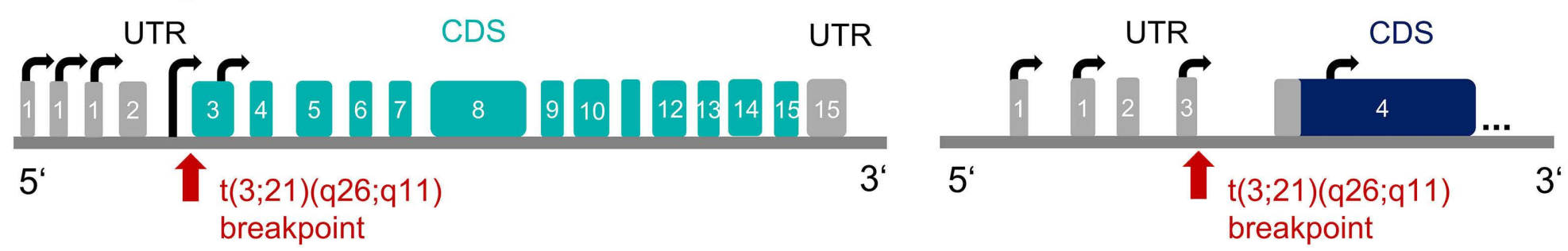

$t(3 ; 21)(q 26 ; q 11)$

NRIP1-EVI1 fusion locus:
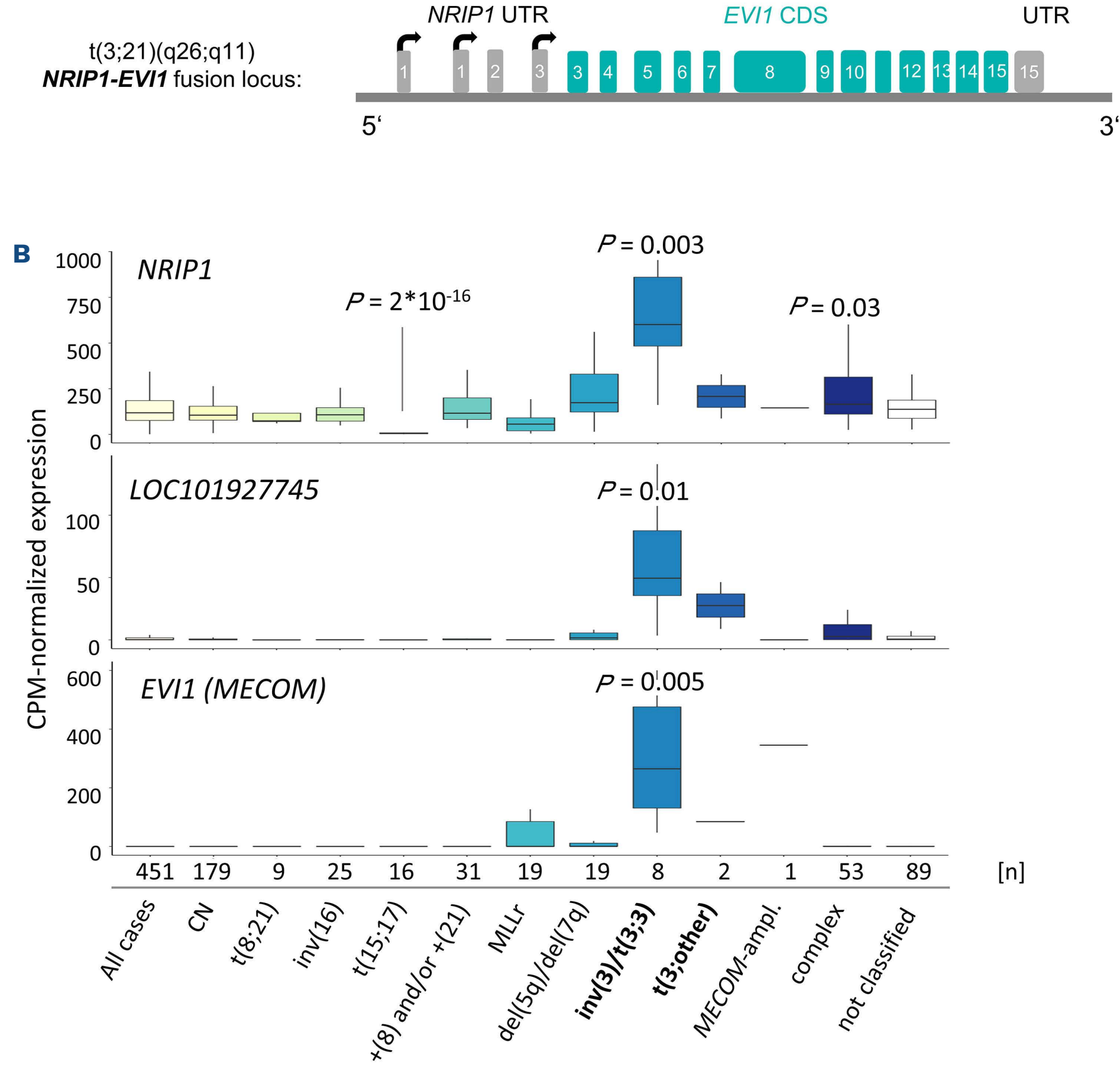

Figure 1. LOC101927745 and NRIP1 are upregulated in EVI1 high acute myeloid leukemia. (A) Schematic representation of the EVI1 and NRIP1 gene loci including the breakpoints and resulting fusion product in $\mathrm{t}(3 ; 21)(\mathrm{q} 26$; q11) cases based on the report by Haferlach et $a l^{8}$ (B) Transcript levels of LOC101927745, NRIP1, and EVI1 (MECOM) in a subset of acute myeloid leukemia patients with recurrent cytogenetic and molecular abnormalities from the Beat AML Master Trial. ${ }^{16}$ Data were TMM-normalized and are presented as counts per million. $P$-values were calculated comparing individual groups to all cases, using the Welch two sample $t$-test with $95 \%$ confidence and adjusted for multiple hypothesis testing. The group $t(3 ;$ other) comprises a $t(2 ; 3)(p 13 ; q 25 ~ 26)$ and a $t(3 ; 11)(p 21.3 ; p 11.2)$ sample. CDS: coding sequence; UTR: untranslated region; CPM: counts per million; CN: cytogenetically normal; MLLr: MLL gene locus rearrangement; TMM: trimmed mean of M values. 
A

Beat AML Master Trial, $n=408$

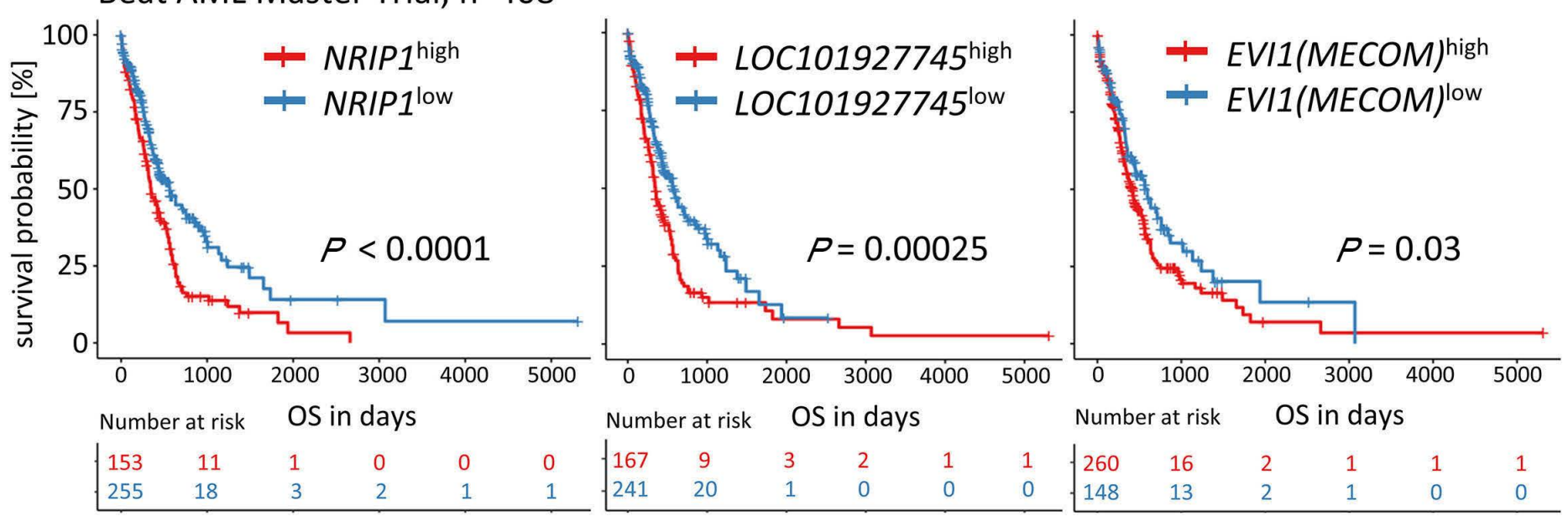

B AMLCG 2008, $\mathrm{n}=249$
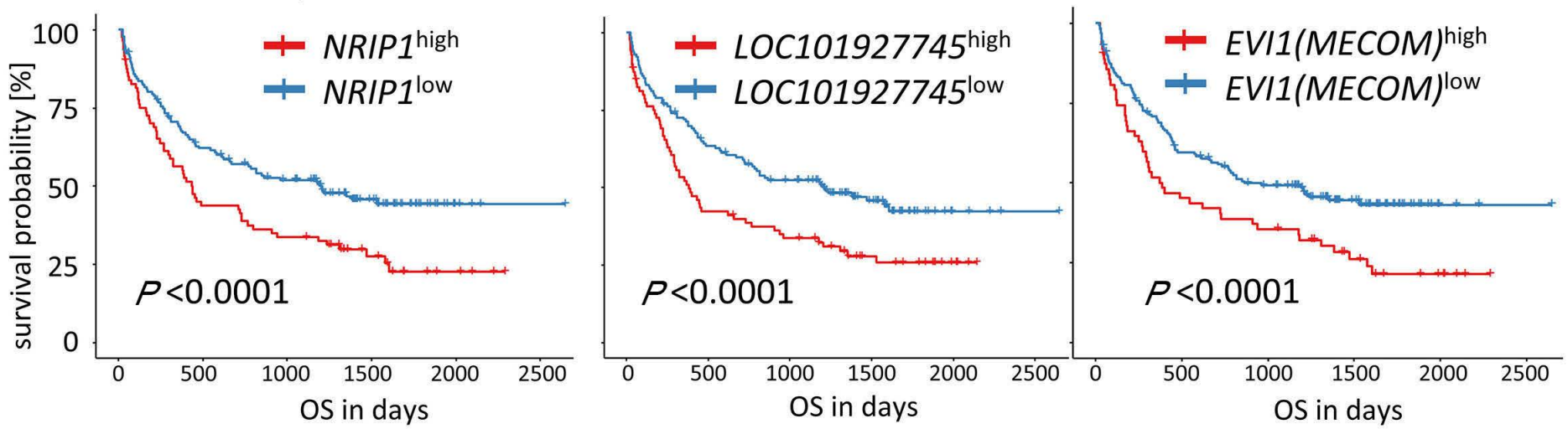

C Complex Kayrotype (CK) AML, $n=33$
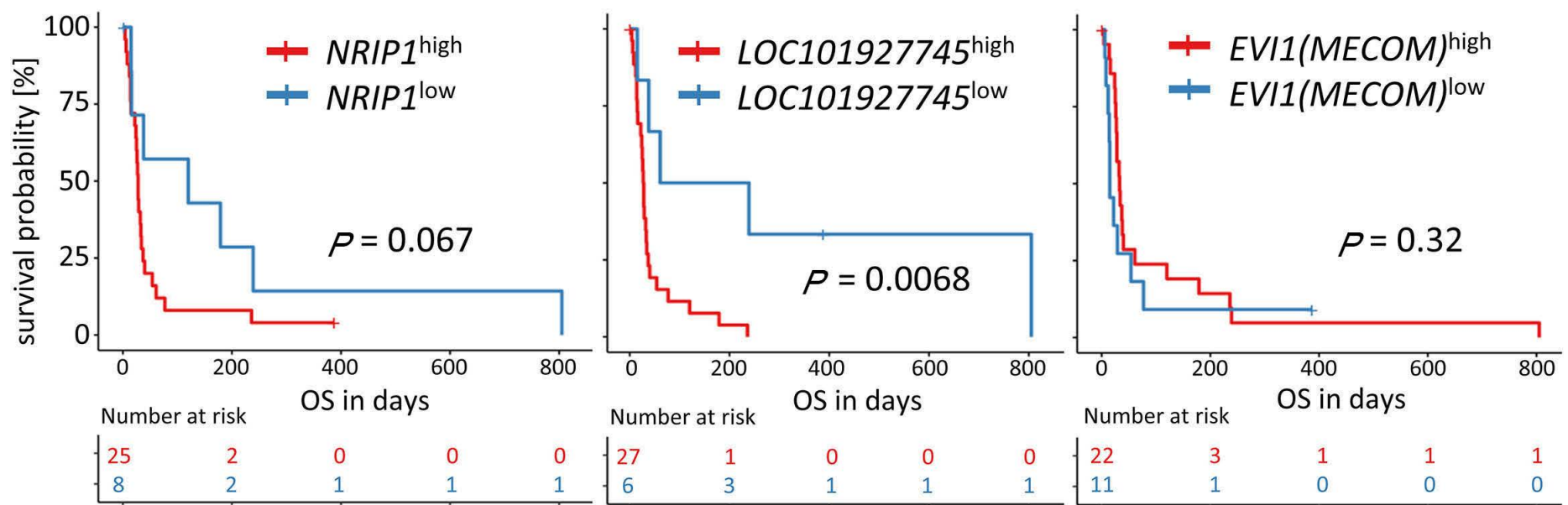

$\mid$\begin{tabular}{ccccc}
27 & 1 & 0 & 0 & 0 \\
6 & 3 & 1 & 1 & 1 \\
\hline
\end{tabular}

D

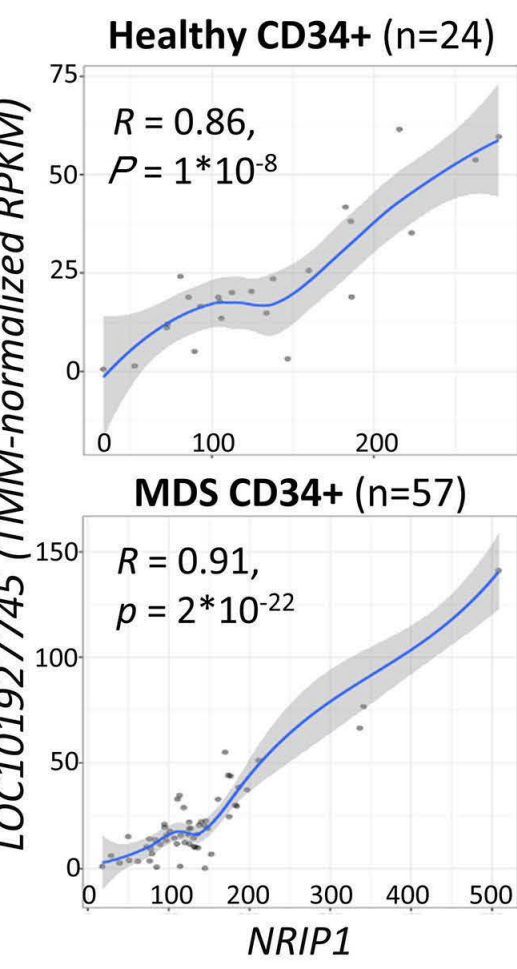

CK-AML ( $n=64)$

\section{E}

AMLCG-2008 $(n=249)$
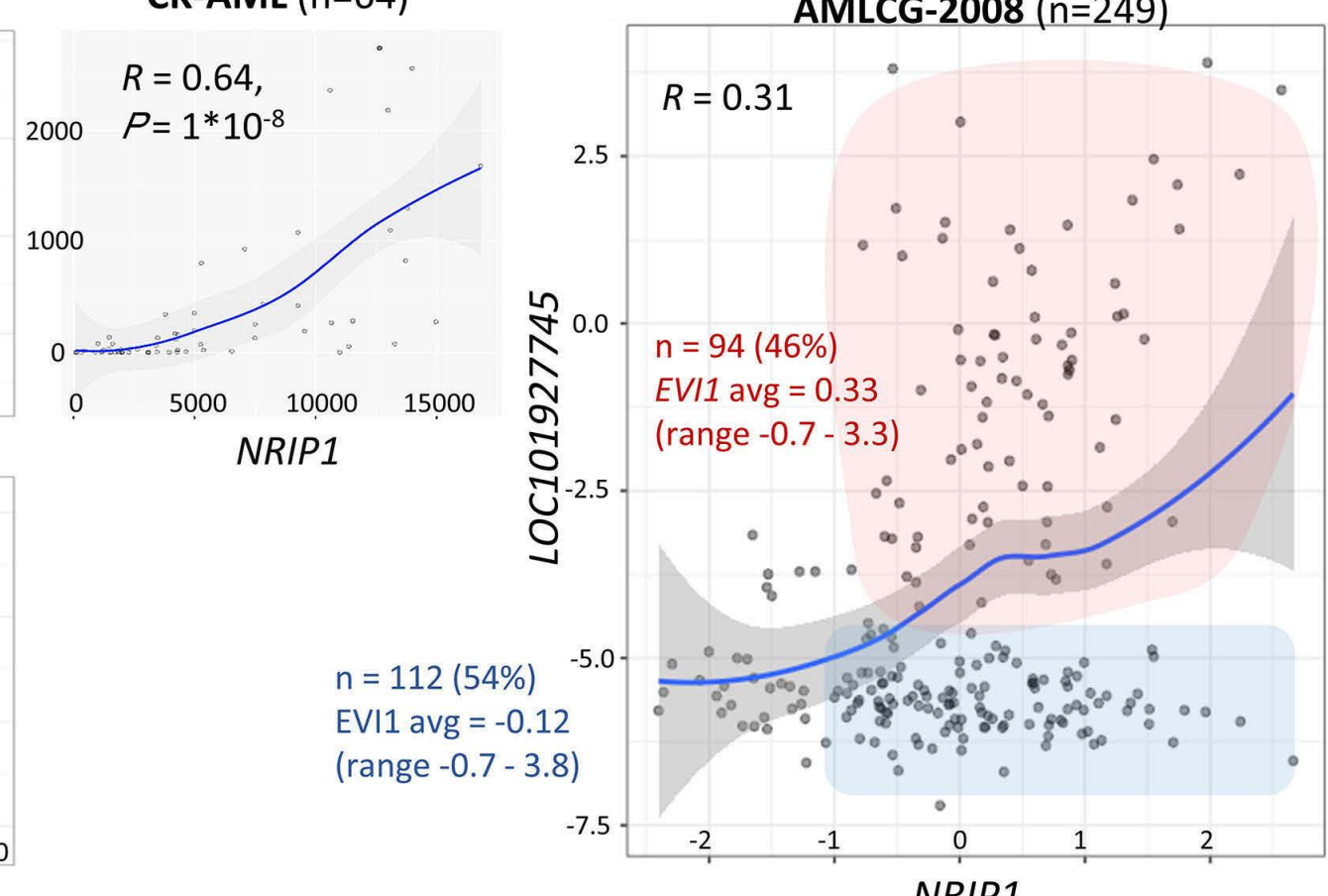

Continued on the following page. 
Figure 2. LOC101927745 and NRIP1 transcript levels are negatively associated with survival in patients with acute myeloid leukemia. (A-C) Graphical representation of Kaplan-Meier estimates based on EVI1 (MECOM), NRIP1 and LOC101927745 expression calculated in: (A) the adult acute myeloid leukemia (AML) subset of the Beat AML Master Trial cohort; ${ }^{16}$ (B) the AMLCG-2008 cohort; ${ }^{19}$ and (C) a subset of patients from the complex karyotype (CK)-AML cohort ${ }^{37}$ for whom survival information was available. $P$-values were calculated using the log-rank test. Patients were dichotomized into groups with high or low expression of the gene of interest using maximally selected rank statistics. ${ }^{34}$ (D) Pairwise correlations of TMM-normalized RNA-sequencing data for LOC101927745 and NRIP1 from healthy CD34+ hematopoietic stem and progenitor cells (GSE114922), primary blast cells from patients with myelodysplastic syndrome (GSE114922) and complex karyotype-AML patients' AML cells ${ }^{37}$ with the respective Pearson $R$, where $R=1$ describes a perfect correlation. (E) Pairwise correlation of TMM-normalized RNA-sequencing data for LOC101927745 and NRIP1 in the AMLCG-2008 cohort with the respective Pearson $R$, where $R=1$ describes a perfect correlation. Data were voom-transformed (variance modeling at the observational level, see Online Supplementary Methods). OS: overall survival; CK: complex karyotype; MDS: myelodysplastic syndrome; TMM: trimmed mean of M values.

with intact chromosome 3 and with low NRIP1 expression, we analyzed public chromatin immunoprecipitation (ChIP)sequencing data assessing TF occupancy and ChIP-sequencing data from AML patients' blasts and cell lines (data from BLUEPRINT ${ }^{17}$ and ENCODE ${ }^{23,24}$ ). NRIP1 low AML cell lines and primary cells displayed a universal lack of TF binding and were devoid of activating histone modifications in the LOC101927745/NRIP1 TAD (data not shown). Instead, these cells displayed an accumulation of repressive histone marks such as H3K27me3 (associated with promotor repression) and $\mathrm{H} 3 \mathrm{~K} 9 \mathrm{me} 3$ (associated with permanent heterochromatin formation) (Online Supplementary Figure S4B). In summary, these findings support our hypothesis that the LOC101927745 genomic site is of relevance for the transcriptional regulation of NRIP1.

\section{NRIP1 transcription is independently regulated by retinoic acid signaling and the GH21J015439 enhancer in acute myeloid leukemia cells}

The promoter sequences of NRIP1 were reported to be rich in RA receptor binding sites. ${ }^{39}$ Another hint towards the relevance of RA signaling for NRIP1 transcription is that $\mathrm{t}(15 ; 17)$ AML patients' samples and the $\mathrm{t}(15 ; 17)$ RA receptor dysfunctional NB-4 cell line do not transcribe NRIP1 at all (Figure 1B, Online Supplementary Figure S1A). Therefore, we aimed to assess whether an external stimulation of RA receptor signaling would induce NRIP1 expression in an NRIP1/ow-expressing AML model. Concurrently, we also aimed to determine whether the deletion of the putative regulatory element GH21J015439, which is embedded within the LOC101927745 TSS, would have an impact on any hypothetical RA-mediated effects on NRIP1 transcription. We, therefore, deleted a 470 bp genomic region spanning GH21J015439, LOC101927745 exon 1, and part of exon 2 (Online Supplementary Figure S4A, C) using a CRISPR/Cas9guided approach in the human OCI-AML5 AML cell line (LOC$\mathrm{KO}$ ), which inherently displays low NRIP1 and lack of LOC101927745 expression (Online Supplementary Figure S1A). Genomic deletion led to a modest but significant reduction of NRIP1 mRNA levels in LOC-KO compared to wild-type OCIAML5 control cells (LOC-WT) $(P=0.05)$ (Figure 4A), confirming that the deleted site likely harbors an NRIP1-enhancing regulatory element. Treatment of both LOC-KO and LOC-WT cell lines with the RA receptor agonist ATRA led to a pronounced upregulation of NRIP1 expression in both cell lines (Figure 4A). As ATRA is known to induce differentiation in AML cells, including OCI-AML5 (Online Supplementary Figure S5), we next tested whether expression of NRIP1 was truly the result of RA receptor signaling or merely a side effect of differentiation. Thus, we independently treated both cell lines with the protein kinase C-activator 12-O-tetra-decanoylphorbol-13-acetate (TPA), which induces differentiation of OCI-AML5 cells in an RA-independent manner. ${ }^{40}$ TPA treatment did not affect NRIP1 transcription, suggesting that NRIP1 was indeed induced by RA receptor signaling and is differentiation independent. Of note, genomic deletion of GH21J015439 also led to a significant reduction of NRIP1 mRNA levels in LOC-KO compared to LOC-WT cells treated with TPA $(P=0.03)$ (Figure $4 A)$ again highlighting the positive regulatory effect of this element on NRIP1 transcription. In addition, LOC-KO cells displayed increased expression of the mature myeloid surface marker CD11c and a reduced proliferation rate compared to LOC-WT cells (Online Supplementary Figure S5B, C).

We were able to confirm the pronounced upregulation of NRIP1 in response to ATRA treatment in an RNA-sequencing dataset generated by Nguyen et al. in MLL-AF9-expressing murine leukemic stem cell-enriched fractions that were treated with $1 \mu \mathrm{M}$ ATRA or control for $24 \mathrm{~h}^{22}$ In these cells, ATRA treatment resulted in an 8.8-fold increase of Nrip1 expression ( $P=0.006)$ (Figure 4B) and, interestingly, ATRA-induced expression of Nrip1 was antagonized by simultaneous shRNA-mediated Evi1-knock-down (7.6-fold increase relative to vehicle-treated sh-control), which reduced Nrip1 levels even more strongly in vehicle controls alone (6.6-fold decrease, $P=0.07$ ) (Figure $4 \mathrm{~B}$ ). This observation hints at a relevance of NRIP1 expression for EVI ${ }^{\text {high }} A M L$ and suggests that NRIP1 could be under the direct transcriptional control of the EVI1 TF complex.

\section{NRIP1 expression is regulated by the oncogenic EVI1 transcription factor network}

To explore whether EVI1 contributes directly to the transcriptional control of NRIP1, we first ensured that there are EVI1 binding sites present within the NRIP1/LOC101927745 TAD and identified 16 sites with a significant z-score that are conserved between human, mouse and rat (Online Supplementary Figure S6). No EVI1 binding motifs were present within 

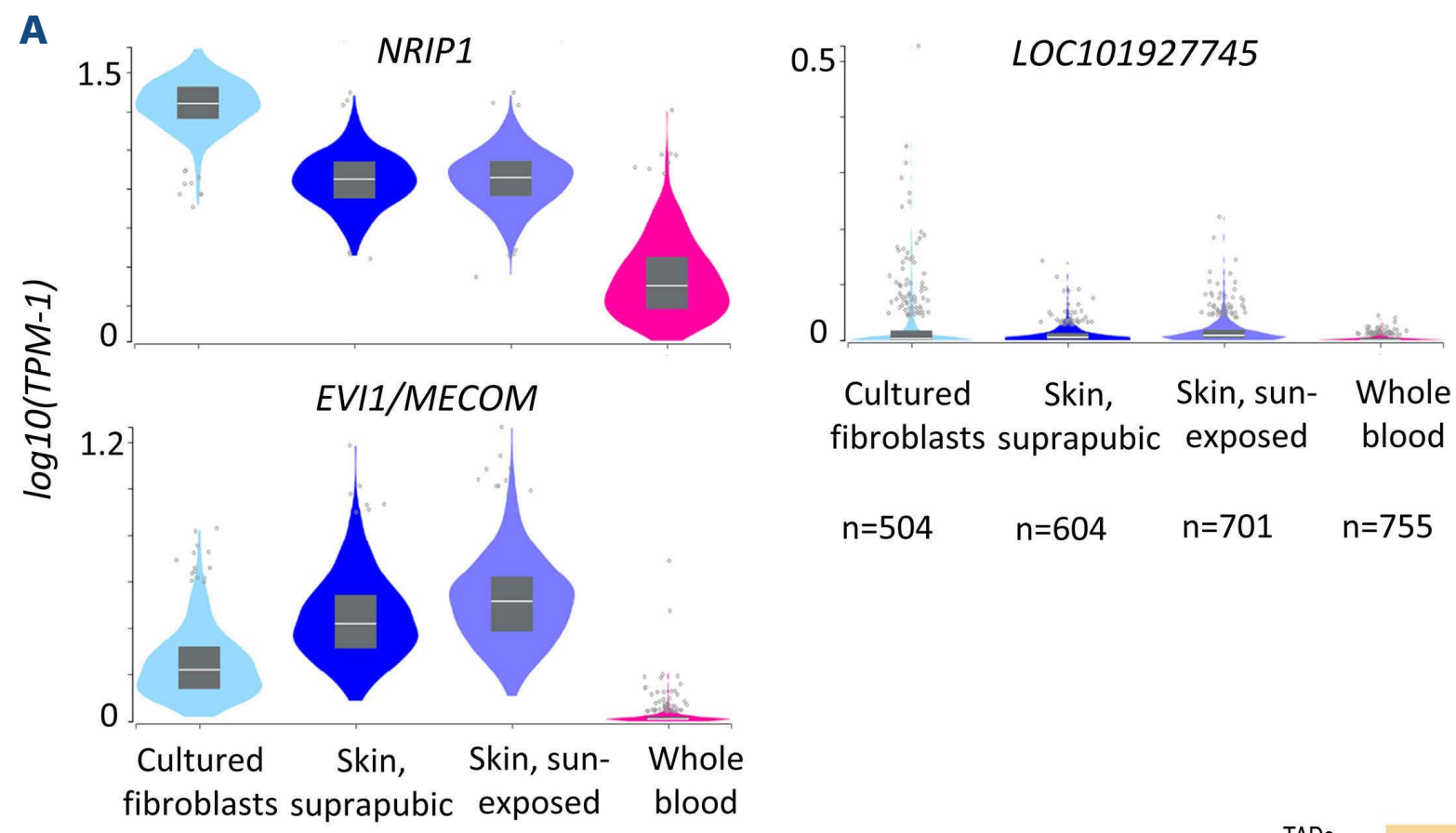

$n=504 \quad n=604 \quad n=701 \quad n=755$

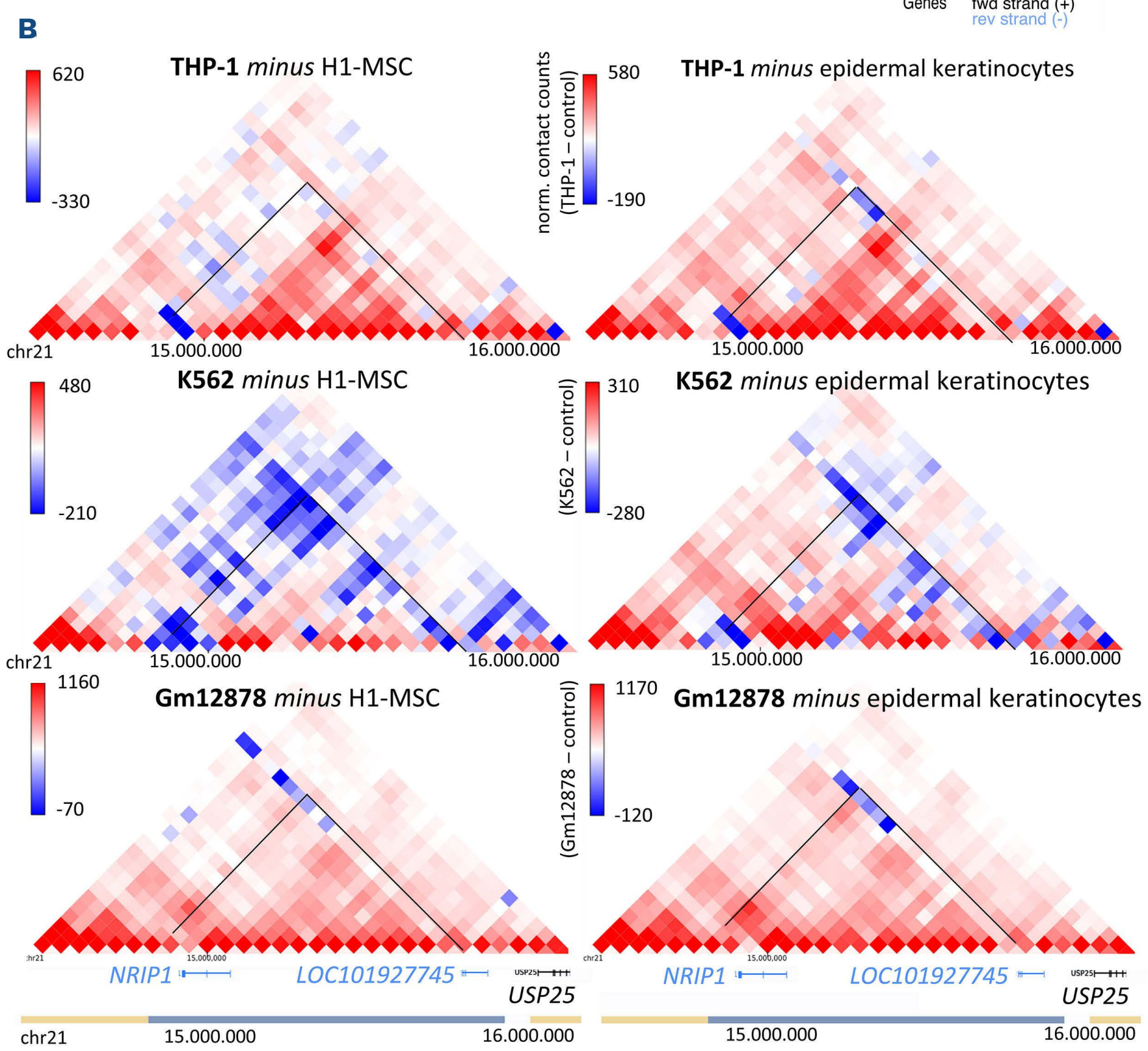

Figure 3. Contact between the LOC101927745 transcription start site and the NRIP1 genomic site is specifically enriched in NRIP1 ${ }^{\text {high }} /$ EVI1 $^{\text {high }}$ tissues. (A) RNA expression of NRIP1, LOC101927745 and EVI1/MECOM in the indicated tissues. Expression data were obtained from the GTEx Portal, dbGaP accession number phs000424.vN.pN in March 2021. (B) Heatmaps generated by subtracting sequencing-based $\mathrm{Hi}-\mathrm{C}$ chromatin contact quantification data which were obtained in NRIP1/igh/EVIhigh skin tissues: human H1 ESC-derived fibroblast-like mesenchymal stromal cells (H1-MSC, left) and human epidermal keratinocytes (right), from Hi-C quantification data obtained in NRIP1/ow/EVI1 negative leukemia cell lines (THP-1, K562, and Gm12878). Blue areas on heatmaps represent sites of high contact in NRIP1 high $/ E V I 1^{\text {high }}$ tissues whereas red areas have greater contact intensities in leukemia cells. The black triangle indicates the region connecting the LOC101927745 transcription start site and the NRIP1 gene

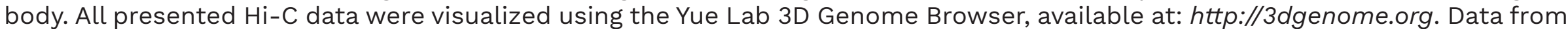
H1-MSC were generated by Dixon et al., ${ }^{30}$ from keratinocytes by Rubin et al., ${ }^{31}$ from THP-1 by Phanstiel et al.. ${ }^{29}$ and from Gm12878 by Rao et $a{ }^{28}{ }^{28}$ TPM: transcripts per kilobase million. 
the NRIP1 promoter but a total of six conserved EVI1 binding sites were present within the LOC101927745 gene, including the putative NRIP1 enhancer GH21J015439, which overlaps the LOC101927745 TSS. To confirm that any of these motifs are actually bound by EVI1 in AML cells, we analyzed ChIP-sequencing data produced by Loke et al. from EVI1-knockdown vs. control SKH-1 AML cells, which harbor a t(3;21)(q26;q22) translocation. The exact $\mathrm{SKH}-1$ breakpoint in chromosome $21 \mathrm{q}$ is located more than $19 \mathrm{Mb}$ upstream of the NRIP1 genomic locus which is therefore retained in this cell line. ${ }^{41}$ Translocation $t(3 ; 21)$ causes expression of a fused RUNX1EVI1 TF, which was found to form an abnormal transcription-
A

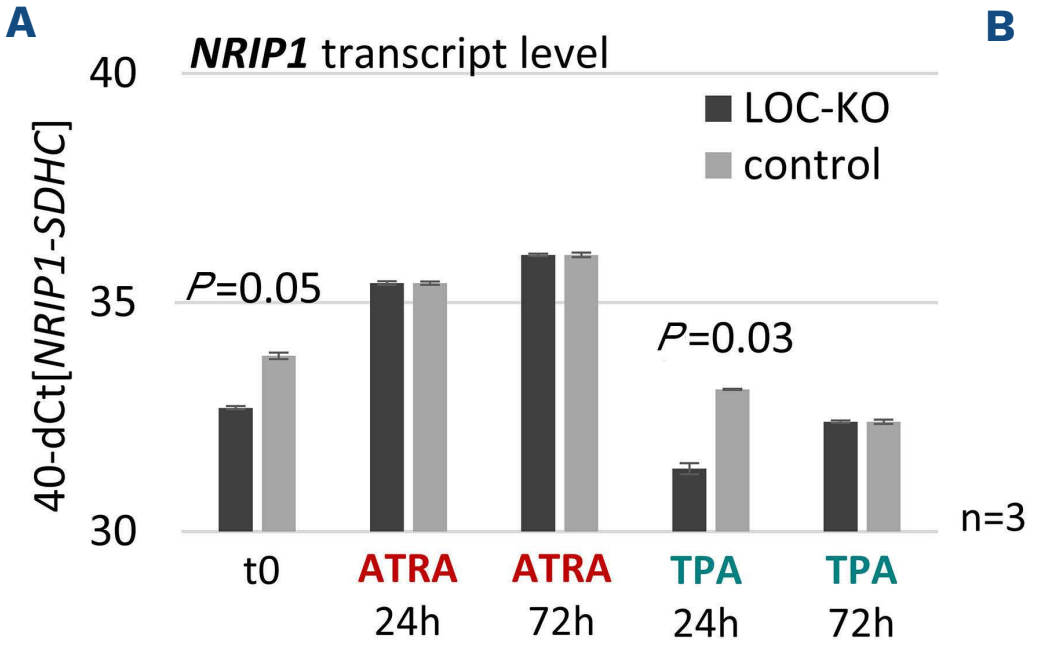

C

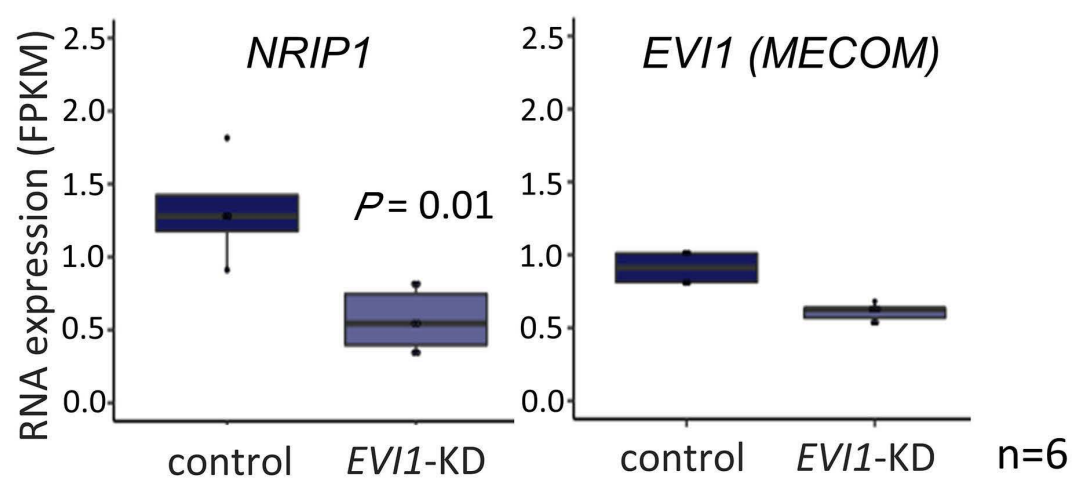

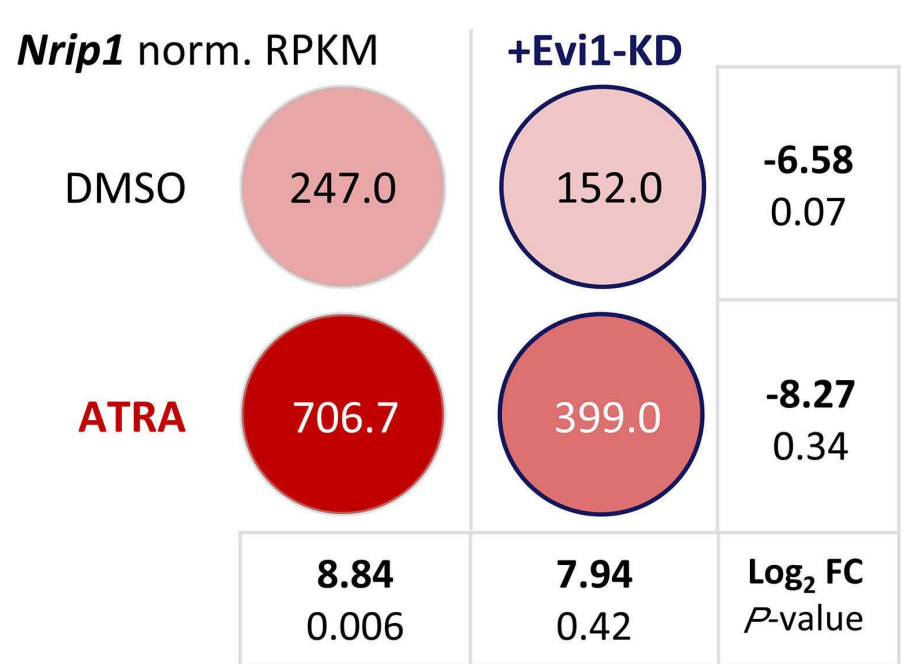

Mecom (Evi1) norm. RPKM

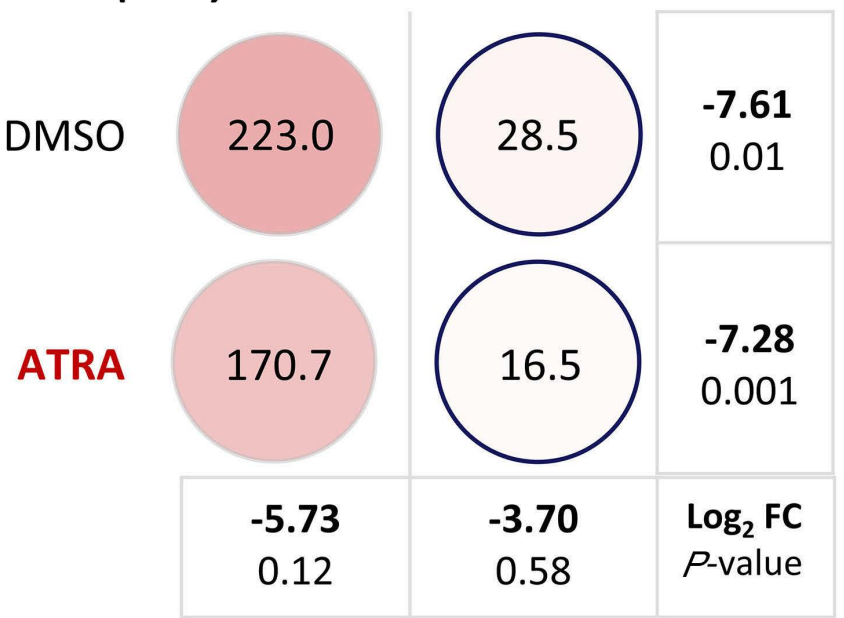

shown are average values from $n=3$

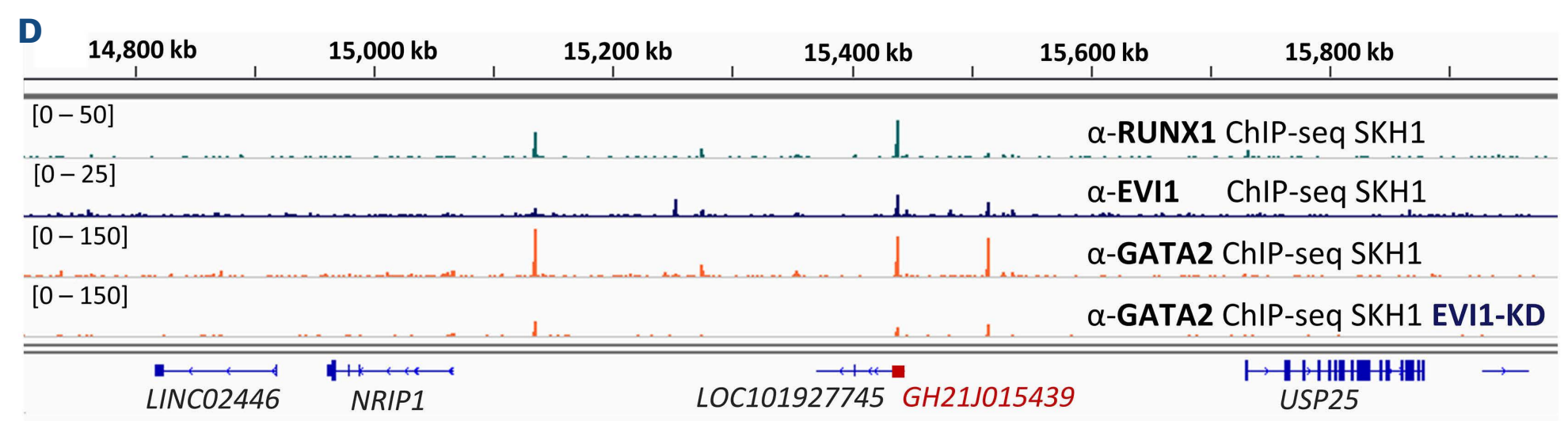

Figure 4. NRIP1 transcription is induced by retinoic acid signaling and reduced upon knockdown of EVI1. (A) Quantitative realtime polymerase chain reaction of NRIP1 in LOC101927745 transcription start site-knockout (LOC-KO) and control OCI-AML5 cells treated with either $0.1 \mu \mathrm{M}$ ATRA or $1 \mathrm{nM}$ TPA for $72 \mathrm{~h}$ in three independent experiments. Total RNA was extracted before, after 24 $\mathrm{h}$ and after $72 \mathrm{~h}$ of treatment, DNase-digested and reverse transcribed. $2^{\text {ddct }}$ was calculated relative to SDHC. $P$-values were calculated using a paired two-tailed Student $t$-test. (B) Normalized RNA-sequencing data for Nrip1 and Mecom, generated by Nguyen et al. ${ }^{22}$ in MLL-AF9-expressing murine leukemic stem enriched cell fractions treated with $1 \mu M$ ATRA or dimethylsulfoxide control for $24 \mathrm{~h}$. (C) Normalized RNA-sequencing data quantifying NRIP1 and EVI1 transcripts, both generated from $\mathrm{t}(3 ; 21)(q 26 ; q 22)$ SKH1 AML cells treated with either EVI1-targeting- or control siRNA. Presented data combined all replicates analyzed $48 \mathrm{~h}(\mathrm{n}=2)$ and $96 \mathrm{~h}(\mathrm{n}=2)$ after siRNA treatment to increase statistical power. Data were generated by Loke et al. ${ }^{26}$ (D) Chromatin immunoprecipitation-sequencing footprinting data for RUNX1, EVI1 and GATA-2, which form an abnormal transcription-activating complex in $\mathrm{t}(3 ; 21) \mathrm{AML}$. Data was generated by Loke et $a l^{26}$ as described in C. $P$-values were calculated using the Welch two sample t-test. LOC-KO: LOC101927745 transcription start site-knockout; ATRA: all-trans retinoic acid; (LOCKO); TPA: 12-O-tetra-decanoylphorbol-13-acetate; FC: fold change; KD: knockdown; DMSO: dimethylsulfoxide; RPKM: reads per kilobase million; FPKM: fragments per kilobase per million. 
activating complex together with GATA-2 and ETS factors. ${ }^{26}$ When analyzing EVI1, RUNX1, and GATA-2 ChIP-sequencing data produced in SKH-1 cells, we found that they displayed strong binding at two EVI1 motifs within the LOC101927745 gene, with the strongest binding of all three TF at the GH21J015439 putative NRIP1 regulatory site as well as high NRIP1 transcript levels in the corresponding RNA-sequencing data (Figure 4C, D). Upon shRNA-mediated EVI1:RUNX1 knockdown, both TF binding and NRIP1 expression were significantly reduced in $\mathrm{SKH}-1$ cells compared to controls (Figure $4 C, D$ ), supporting our hypothesis that the NRIP1 gene is under the direct control of the abnormal EVI1 TF complex that drives this AML phenotype.

Assuming that LOC101927745 upregulation is indeed controlled by an oncogenic EVI1 TF complex, LOC101927745 and EVI1 transcript levels would be expected to correlate in patients' samples. Indeed, similar to our observations regarding a correlation between LOC101927745 and NRIP1 RNA, transcript levels of LOC101927745 and EVI1 showed a high degree of correlation in normal and MDS hematopoietic stem and progenitor cells ( $R=0.87$ ) (Figure $5 \mathrm{~A}$ ). According to AML data, $2.9 \%$ to $10.5 \%$ of all patients expressed both transcripts $\left(\mathrm{LOC}^{+} / E V I 1^{+}\right), 66.9 \%$ to $78 \%$ expressed neither ( $\left.\mathrm{LOC}^{-} / \mathrm{EVII}^{-}\right)$, $3.4 \%$ to $8.7 \%$ expressed only EVI1 in the absence of LOC101927745 (LOC $/$ LVVI $\left.^{+}\right)$and $13.9 \%$ to $15.7 \%$ of patients only expressed LOC101927745 (LOC $\left.{ }^{+} / E V I 1^{-}\right)$, suggesting that LOC101927745 and therefore NRIP1 transcription is not exclusively regulated through EVI1 in AML patients (Figure 5A). When comparing survival among these four groups, patients who expressed LOC101927745 RNA had significantly worse outcome or response to treatment, independently of EVI1 expression ( $P=0.04$ and 0.0018 in the AMLCG-2008 and Beat $A M L$ cohort, respectively) (Figure 5B). Of note, expression of the LOC101927745 transcript was able to further sub-stratify adverse-risk EVI7 ${ }^{\text {high }} \mathrm{AML}$ patients, highlighting cases with especially poor outcome.

\section{NRIP1 knockdown affects proliferation, viability, and response to all-trans retinoic acid in chromosome 3 rearranged acute myeloid leukemia cells}

As NRIP1 is strongly upregulated in AML cases with chromosome $3 q$ rearrangements, we further assessed the dependence of EVI1-expressing AML blasts on NRIP1 expression. Therefore, we performed both transient antisense- and stably integrated shRNA-mediated knockdown of NRIP1 in $E V I 1^{\text {high }}$ and EVI1 negative $A M L$ cell lines with NRIP1 expression. Stable NRIP1 knockdown significantly affected growth and viability in $\mathrm{t}(3 ; 3)$ UCSD-AML1 and HNT-34 (Figure 6A, B) but to a lesser extent or not at all in chromosome 3 intact $\mathrm{OCl}$ AML3, Kasumi-1, and K562 cells (Online Supplementary Figure $S 7 A, B)$. Knockdown of NRIP1 was confirmed at RNA and protein levels in all cell lines (Figure 6C, D, Online Supplementary Figures S7C, S8 and S9). NRIP1 knockdown rendered t(3;3) cells significantly more sensitive to ATRA treatment, as exemplified by decreased proliferation (HNT-34 88\% and UCSD-AML1 39\% reduction) (Figure 6A) and higher levels of apoptosis after $72 \mathrm{~h}$ of treatment compared to controls (HNT-34 46\% and UCSD-AML1 37\% increase in apoptotic cells) (Figure 6B). In line with our earlier findings in OCl-AML5 cells, ATRA treatment induced expression of NRIP1 and, of note, also resulted in increased EVI1 transcription in $\mathrm{t}(3 ; 3)$ cells as well as in the EVI1-negative cell line OCI-AML3 (Online Supplementary Figures S10 and S11). Furthermore, NRIP1knockdown resulted in significantly elevated transcription of LOC101927745 RNA exclusively in $\mathrm{t}(3 ; 3)$ cells (Figure 6C, Online Supplementary Figure S10). In all EVI1-expressing cell lines, including chromosome 3 normal K562 cells, NRIP1-knockdown resulted in reduced EVI1 transcription (Figure 6C, Online Supplementary Figure S7). Proliferation and apoptosis data recorded in $\mathrm{t}(3 ; 3)$ UCSD-AML1 and HNT-34 cells as well as in chromosome 3 normal OCl-AML3 and NRIP1 ${ }^{\text {negative }}$ NB-4 cells after transient transfection with NRIP1-targeting GapmeRs, confirmed our observation that cell lines with chromosome $3 q$ rearrangements were more vulnerable to NRIP1-knockdown, especially in combination with ATRA treatment (Online Supplementary Figure S11A-C). In contrast, GapmeR-mediated knockdown of LOC101927745 RNA in UCSD-AML1 did not affect RIP1 transcription or proliferation and proliferation and cell viability, supporting a regulatory model in which the LOC101927745 genomic site - i.e., its function as an enhancer - but not its RNA transcript, is relevant for the control of NRIP1 expression (Online Supplementary Figure S11D).

\section{Discussion}

In a large collection of transcriptomic and epigenomic datasets, we found that expression of LOC101927745 and NRIP1 on chromosome 21 is markedly upregulated in AML patients with chromosome $3 q$ abnormalities. Although the majority of AML patients do not express LOC101927745 at comparably high levels, overall between $18.6 \%$ and $24.4 \%$ of AML cases had detectable LOC101927745 transcription in the Beat AML and AMLCG-2008 cohorts, and this was associated with especially poor outcome in AML and was independent of EVI1 transcription status.

In all the datasets we studied, we found that whenever LOC101927745 is transcribed, so too is NRIP1 and that their expression correlates significantly. Based on our findings, we propose the following functional interaction model between NRIP1 and LOC101927745 in AML: blast cells that do not express either LOC101927745 or NRIP1 display a repressive heterochromatin state in the LOC101927745/NRIP1 TAD (Figure 7A). In EVI ${ }^{\text {high }}$ MDS and AML cells, NRIP1 transcription is regulated predominantly through usage of the GH21J015439 enhancer site, which is embedded within the LOC101927745 TSS. Binding of the enhancer is at least in part mediated by the 
A
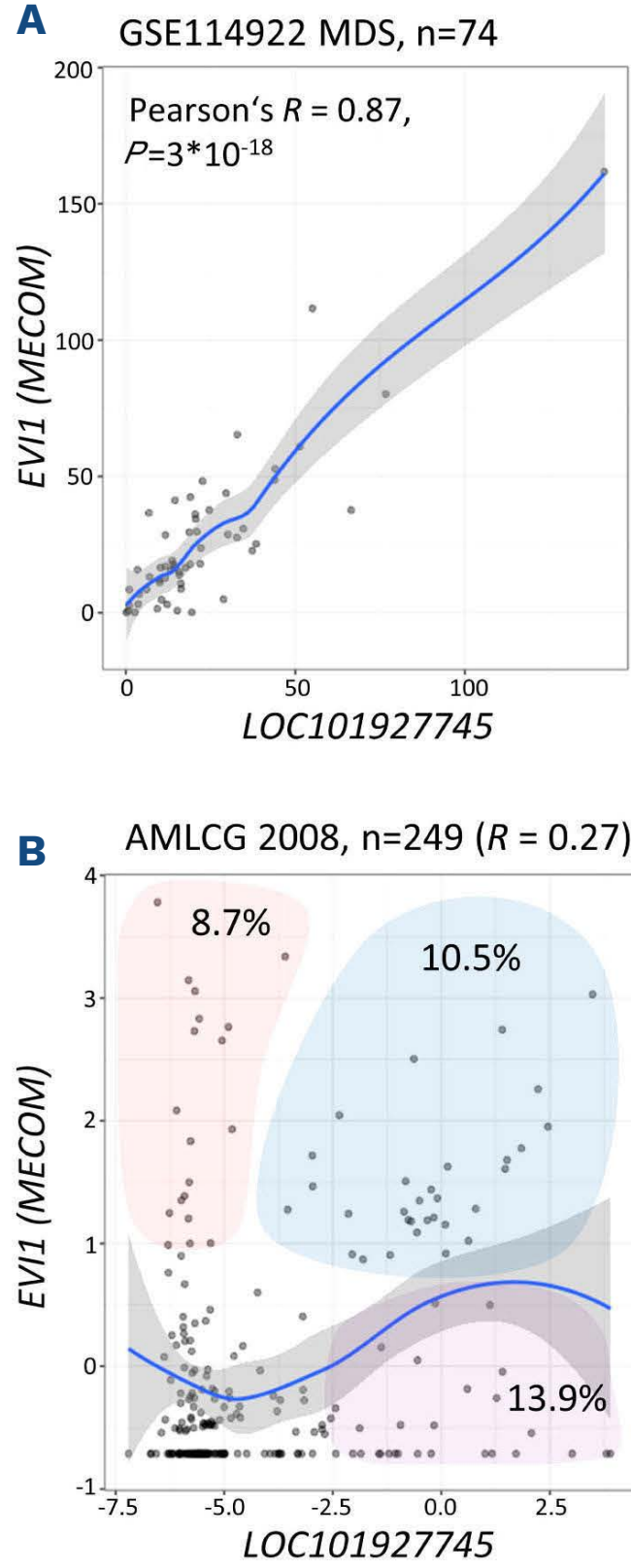

Beat AML Trial, $\mathrm{n}=408(R=0.38)$

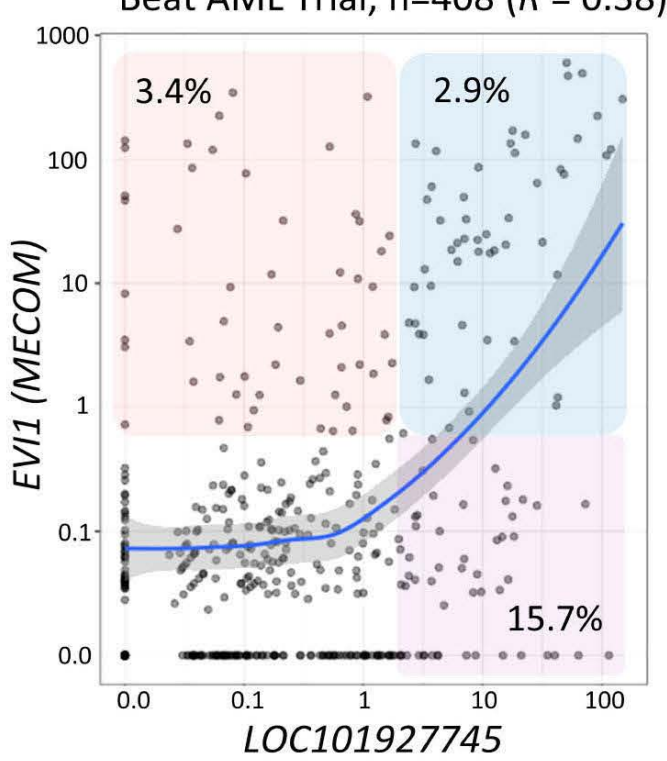

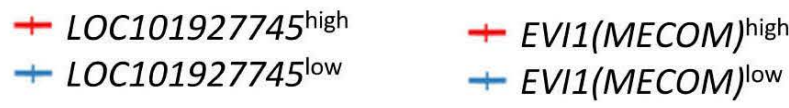
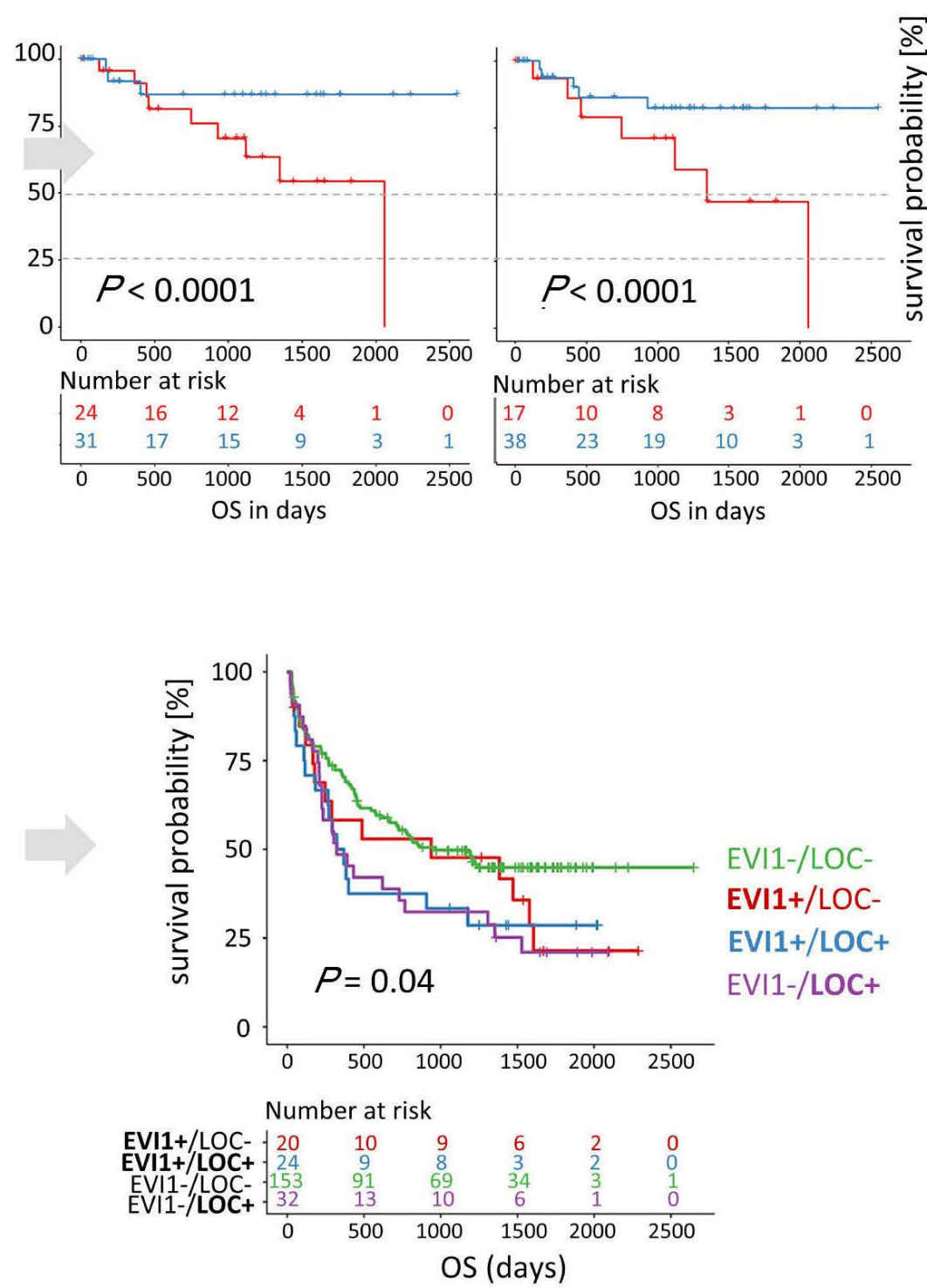

웅
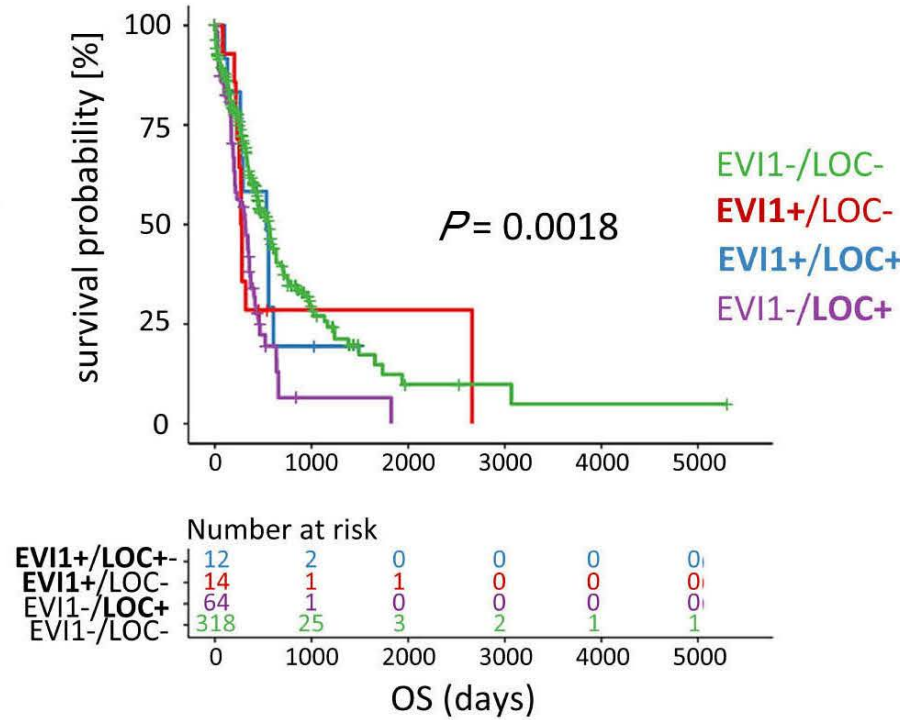

Figure 5. Transcription of LOC101927745 is associated with negative outcomes independently of EVI1 status in patients with acute myeloid leukemia. (A) Left: pairwise correlations of TMM-normalized RNA-sequencing data for EVI1 (MECOM) and LOC101927745 in primary blast cells from patients with myelodysplastic syndrome (GSE114922) with the respective Pearson $R$, where $R=1$ describes a perfect correlation. Right: Kaplan-Meier plots based on LOC101927745 and EVI1 (MECOM) expression calculated in the subset of patients with available survival information. (B) Left: pairwise correlations of TMM-normalized RNA-sequencing data for EVI1 (MECOM) and LOC101927745 from the AMLCG-2008 cohort ${ }^{19}$ with respective Pearson R. Data were voom-transformed (variance modeling at the observational level, see Online Supplementary Methods). Right: Kaplan-Meier plot based on LOC101927745 and EVI1 (MECOM) transcript levels stratified as indicated by the color coding in the subset of patients with available survival information of the same dataset. (C) Left: pairwise correlations of TMM-normalized RNA-sequencing data for EVI1 (MECOM) and LOC101927745 from the Beat AML trial ${ }^{16}$ with the respective Pearson $R$. Axes are log ${ }_{10}$ transformed. Right: Kaplan-Meier plot based on LOC101927745 and EVI1 (MECOM) expression stratified as indicated by the colour coding in the subset of patients with available survival information of the same dataset. MDS: myelodysplastic syndrome; TMM: trimmed mean of M values; OS: overall survival. 
A

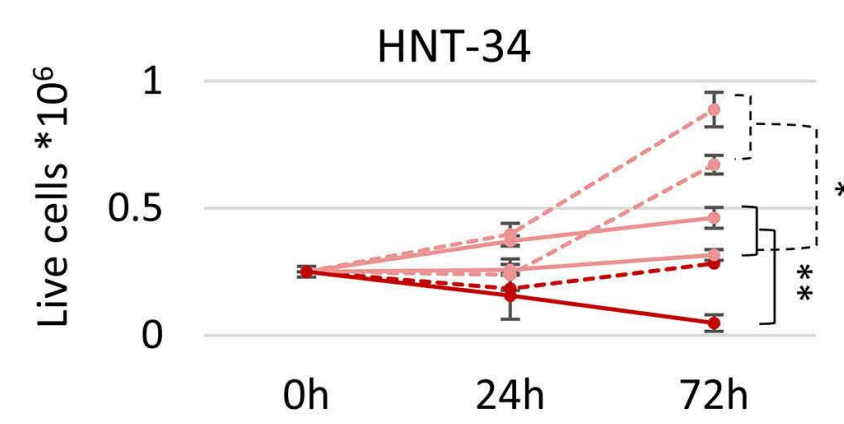

B

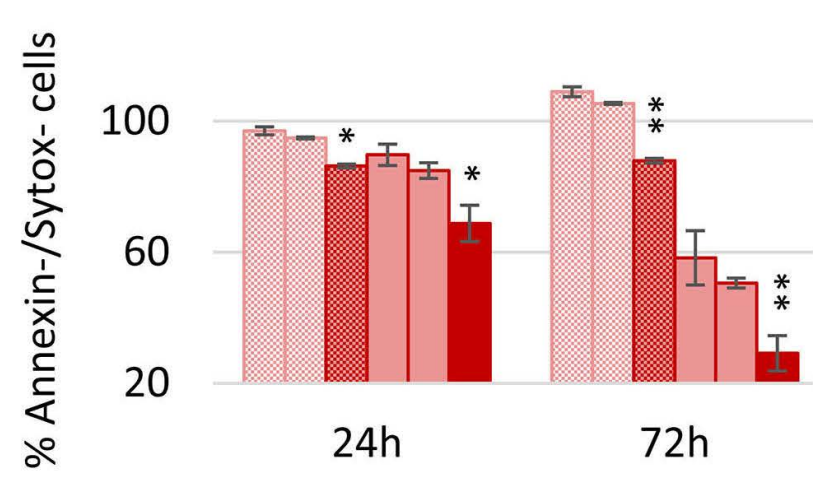

C

1.5

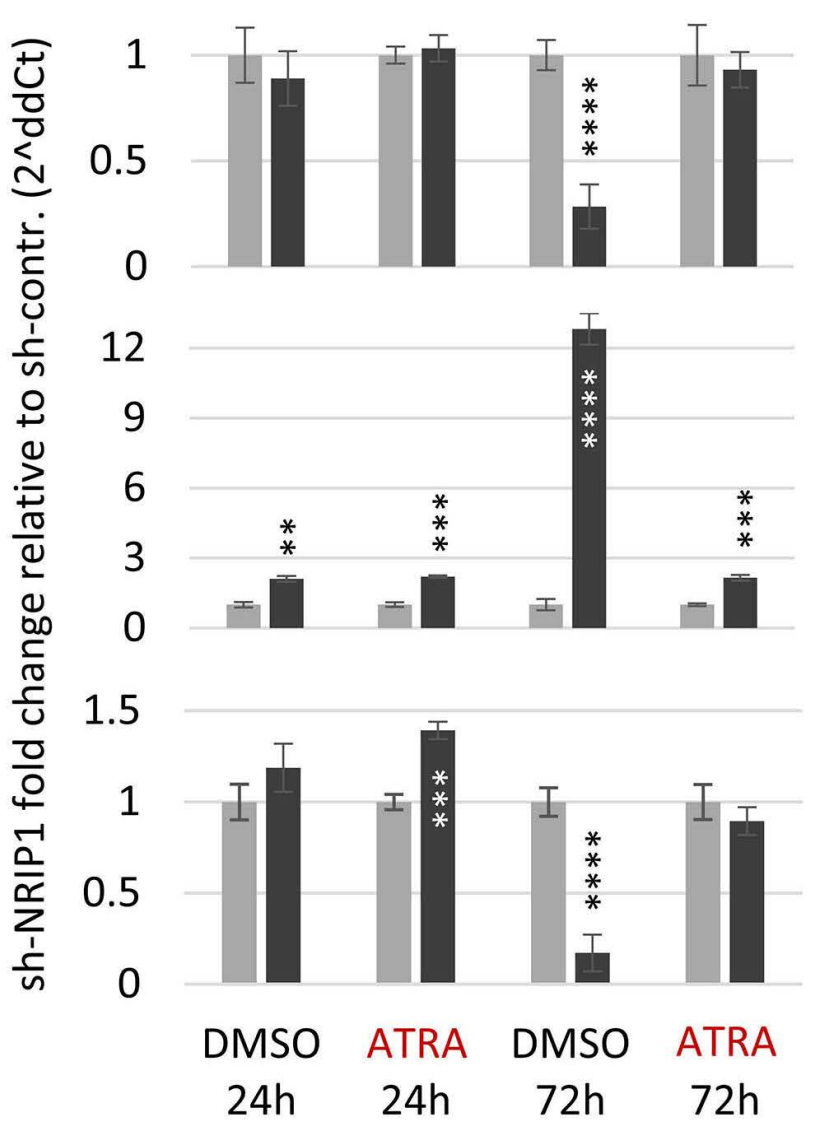

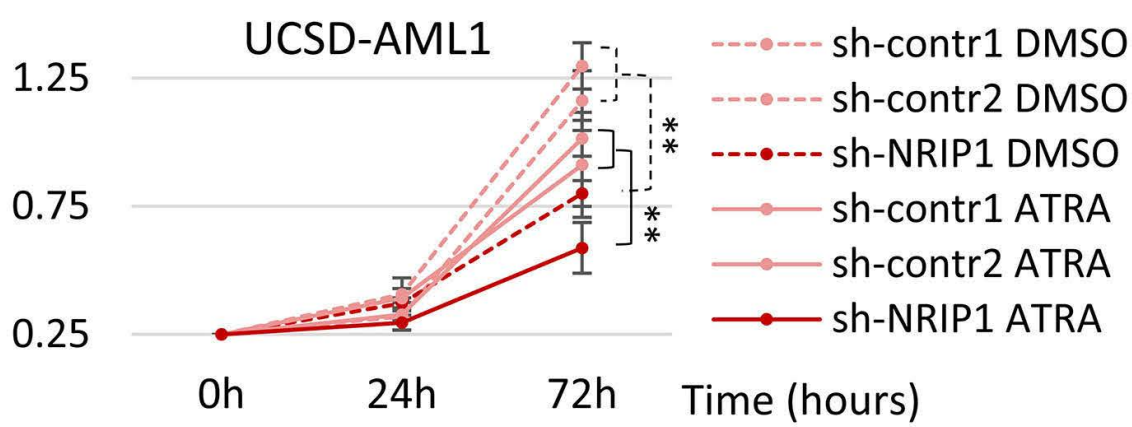

100

60

20

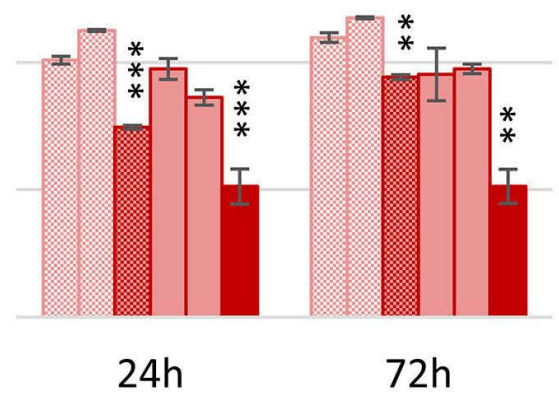

sh-contr 1 DMSO

sh-contr 2 DMSO

圆 sh-NRIP1 DMSO

$\square$ sh-contr 1 ATRA

$\square$ sh-contr 2 ATRA

sh-NRIP1 ATRA

Time (hours)

1.5

UCSD-AML1

sh-contr. $(\mathrm{n}=6)$

a sh-NRIP1 ( $n=3$ )

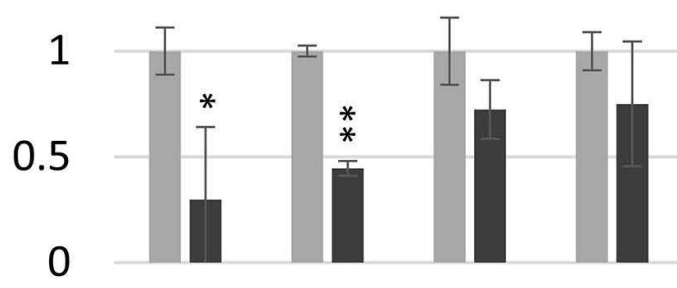

NRIP1

\section{LOC101927745}

exon 1

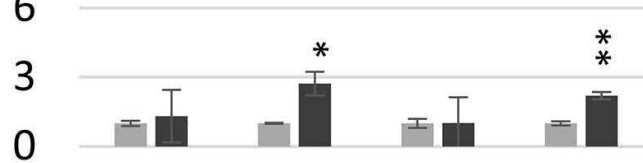

1.5

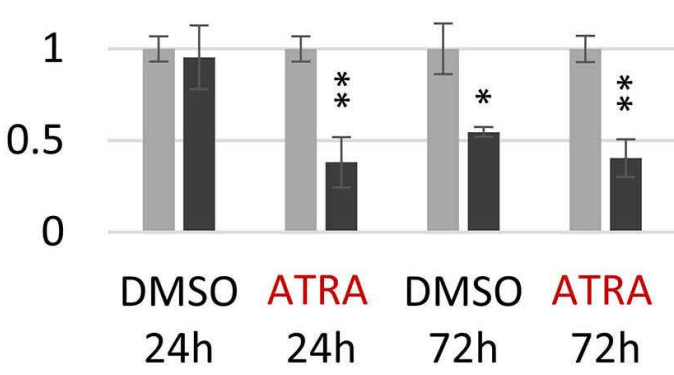

MECOM (EVI1)

D

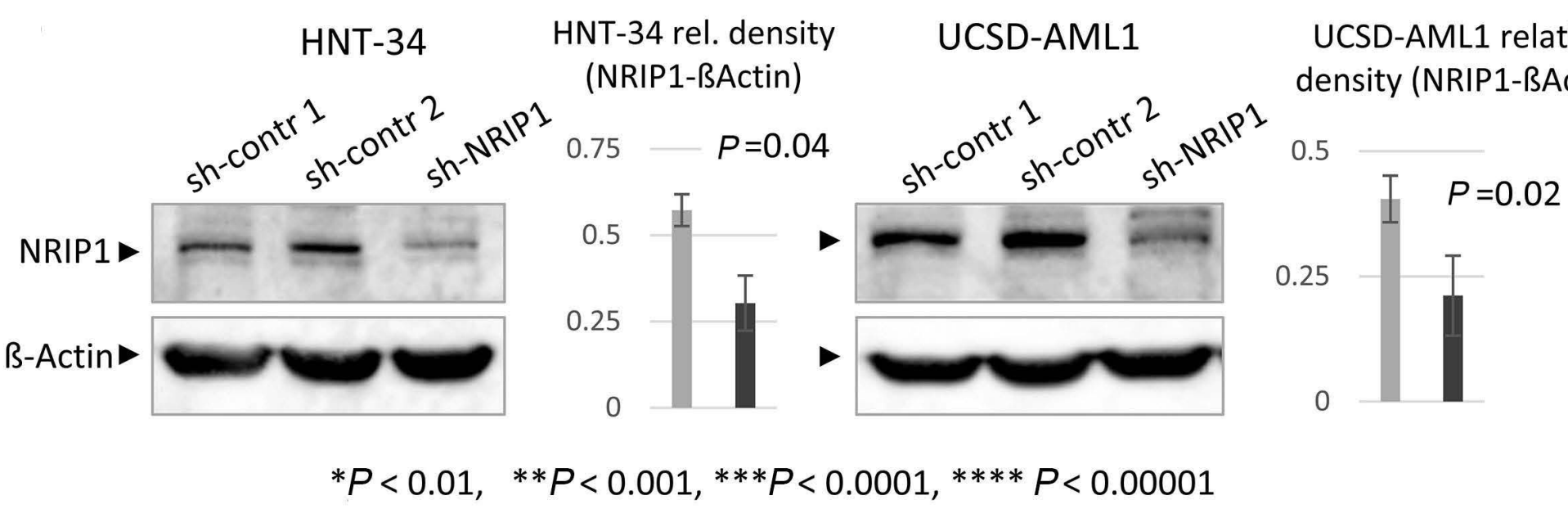


Figure 6. Knockdown of NRIP1 induces apoptosis and increases sensitivity to all-trans retinoic acid in $\mathbf{t}(3 ; 3)$ acute myeloid leukemia cells. (A, B) t $(3 ; 3)$ HNT-34 and UCSD-AML1 cells were lentivirally transduced with constructs encoding either NRIP1targeting or two different control shRNA in three independent experiments. Puromycin-selected cells were seeded at $0.25 \times 10^{6}$ cells $/ \mathrm{mL}$ and treated with with either $0.5 \mu \mathrm{M}$ all-trans retinoic acid or dimethylsulfoxide control and a total of three replicates per condition were harvested and analyzed after $24 \mathrm{~h}$ and $72 \mathrm{~h}$. (A) Cells were resuspended, stained with trypan blue and counted at the indicated time points. The figure shows the total number of trypan-negative (live) cells per well over time. (B) Cells were stained with annexin-V-APC and Sytox viability dye at the indicated time points and 10,000 cells per sample were recorded via flow cytometry. The figure shows the percentage of annexin/Sytox double negative (i.e., non-apoptotic) cells relative to day 0 of the experiment. (C) RNA was extracted from three individual treatment samples per condition, DNase-digested and reverse-transcribed to cDNA. In the same sample, RNA levels of NRIP1, EVI1 (MECOM), and the housekeeping gene ABL1 were quantified using commercially available TaqMan assays in technical triplicates. Transcript levels of LOC101927745 (exon 1) were quantified using SYBR Green chemistry and compared to the predetermined levels of the housekeeping gene SDHC. Fold changes were calculated using the $2^{\text {ddct }}$ method with sh-contr. samples set to 1. (D) Total protein was extracted, BSA-quantified and western blots were performed with NRIP1- and $\beta$-actin targeting antibodies from three individually generated cell lines per condition. The signal intensity of NRIP1 relative to $\beta$-actin bands was quantified using Fiji/Image J. All $P$-values shown were calculated using paired, two-tailed Student $t$ tests for unequal variance. ATRA: all-trans retinoic acid; DMSO: dimethylsulfoxide.

A

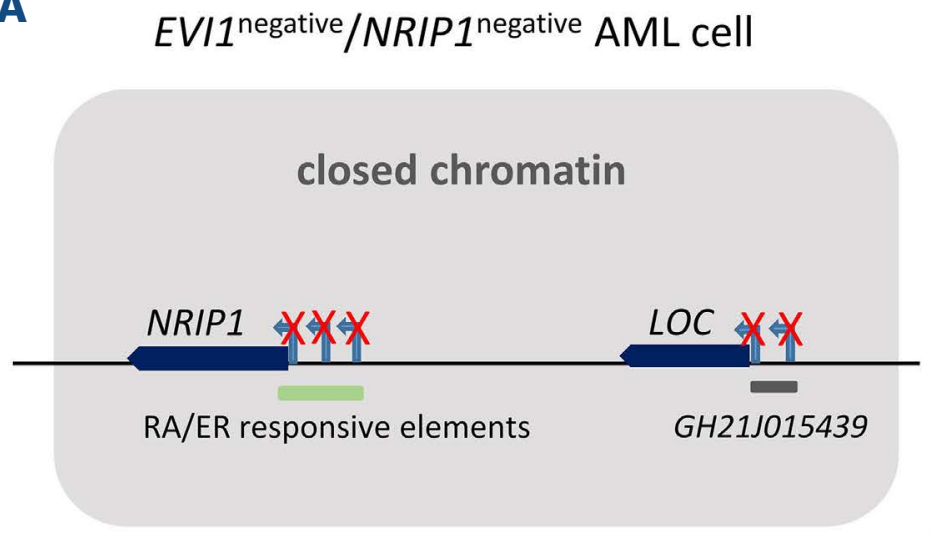

C

EVI1 ${ }^{\text {negative }} /$ NRIP1 $1^{\text {low-high }} \mathrm{AML}$ cell
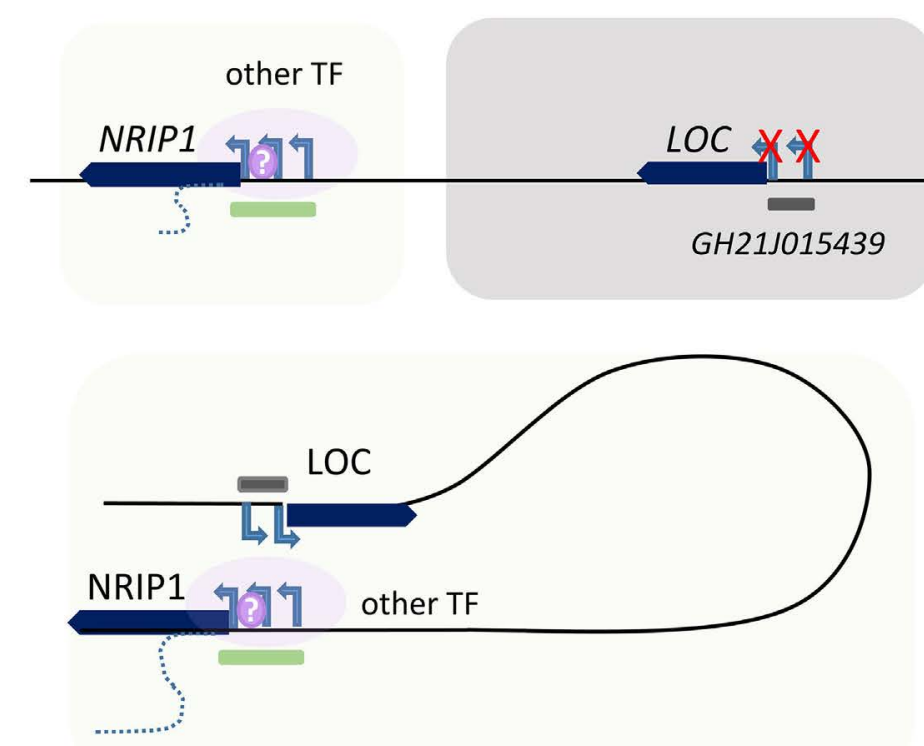

B

EVII ${ }^{\text {high }} \mathrm{AML}$ cell

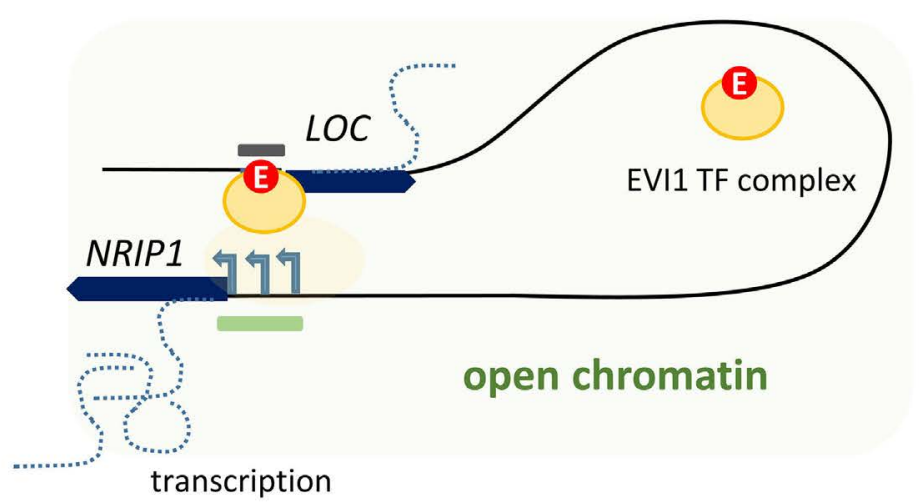

D EVII low AML cell with LOC-KO +/- ATRA
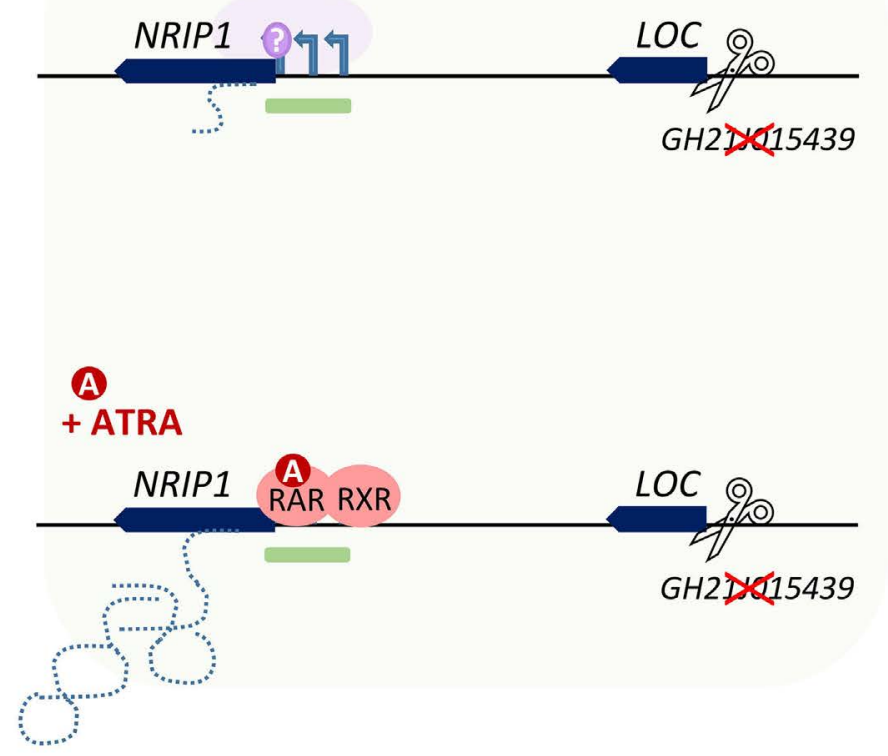

Figure 7. Schematic model of NRIP1 transcriptional regulation in acute myeloid leukemia. (A) Cells that neither express $L O C 101927745$ nor NRIP1 display tightly coiled heterochromatin that is marked by repressive histone modifications and is inaccessible to transcription factors (TF). (B) In EVI1-expressing acute myeloid leukemia (AML) cells, the oncogenic EVI1 TF complex binds the GH21J015439 enhancer located in the LOC101927745 transcription start site, which causes the enhancer complex to loop onto the NRIP1 promoter sites. This process delivers the RNA transcription machinery to the site of contact, resulting in simultaneous transcription of the LOC101927745 and NRIP1 genes. (C) In NRIP1-expressing cells that do not express the EVI1 TF, the NRIP1 promoters are bound and transcription is activated directly, without the involvement of GH21J015439, by other TF (as was shown for retinoic acid- and estrogen receptors). Top: while the NRIP1 gene locus is accessible, the LOC101927745 genomic site is in a repressed, inaccessible state. Bottom: an alternative mode of NRIP1 transcriptional activation in EVI1 negative /NRIP1/ow-high cells involves binding of TF other than EVI1 to the GH21J015439 enhancer promoting NRIP1 transcription. (D) CRISPR-Cas9-mediated excision of GH21J015439 (LOC-KO) in EVI 1 negative/NRIP1 low expressing AML cells only modestly affected NRIP1 transcription and did not prevent promotermediated activation of the NRIP1 gene through retinoic acid receptor (RAR):retinoid X receptor (RXR) heterodimers, which were induced via external stimulation with all-trans retinoic acid. AML: acute myeloid leukemia; RA: retinoic acid; ER: estrogen receptor; ATRA: all-trans retinoic acid; TF: transcription factor; RAR: retinoic acid receptor; RXR: retinoid X receptor. 
EVI1 oncogenic TF complex, which does not bind the NRIP1 promoter directly but instead forms a looped chromatin contact as is common for enhancer:promoter interactions ${ }^{42}$ (Figure 7B). This process recruits the transcriptional machinery to the site of contact which results in simultaneous transcription of both the LOC101927745 and NRIP1 genes. Hence, the expression of the LOC101927745 transcript, regardless of its functionality, is indicative of the accessibility and activation of the NRIP1 enhancer GH21J015439. LOC101927745 and EVI1 transcript levels correlate significantly in EVI1 ${ }^{\text {high }}$ healthy and MDS CD34 ${ }^{+}$cells. Thus, NRIP1 transcriptional control through usage of the GH21J015439 enhancer might reflect how NRIP1 expression is physiologically regulated in immature hematopoietic cells and in other EVI1-expressing tissues such as fibroblasts. In more mature cells and in about $50 \%$ of NRIP1-expressing AML patients, NRIP1 transcription is activated via additional mechanisms that are not mediated through GH21J015439 and therefore do not cause LOC101927745 transcription (Figure 7C). Comparing survival of AML patients stratified according to their expression patterns of NRIP1, EVI1 and LOC101927745, we found that transcription of LOC101927745 was the most reliable prognostic factor among all groupings and that it was highly associated with a dismal overall outcome in the presence or absence of EVII transcription.

Due to the high degree of functional redundancy in the regulatory landscape of physiologically relevant genes, deletion of a single enhancer site is not necessarily expected to have an impact on gene expression as this greatly depends on the presence of transcriptional regulators such as TF and histone modifiers. Nevertheless, we observed a modest but significant reduction of NRIP1 transcript levels after excising GH21J015439 in an NRIP1 ${ }^{\text {low }}$-expressing AML cell line, which enhanced its response to differentiation-inducing drugs. However, removal of this enhancer site did not hinder the transcriptional upregulation of NRIP1 through ATRA-stimulated RA signaling (Figure 7D), which we have identified to serve as an independent mechanism that induces NRIP1 expression in human AML cells.

Utilizing two different knockdown strategies in combination with ATRA-mediated induction of NRIP1 transcription, we observed that a forced downregulation of NRIP1 is harmful to $\mathrm{t}(3 ; 3) E V I 1^{\text {high }}$ AML cells. Furthermore, NRIP1-knockdown rendered chromosome 3 rearranged cell lines vulnerable to ATRA treatment, resulting in decreased growth and induction of apoptosis. After $72 \mathrm{~h}$ of ATRA treatment, we detected an upregulation of EVI1 in $\mathrm{t}(3 ; 3)$ and, surprisingly, in the chromosome 3 normal cell line OCI-AML3. Increased EVI1 expression also resulted in an upregulation of LOC101927745 RNA in t(3;3) cells which, according to our proposed model of NRIP1 regulation in $E V I 1^{\text {high }} A M L$, likely reflected increased enhancer usage in an attempt to upregulate NRIP1. In line with the findings of a study by Nguyen et al., ${ }^{22}$ reporting that ATRA enhances the oncogenic effects of EVI1, these observations highlight the relevance of NRIP1 for EVI1-expressing AML cells and suggest that NRIP1 might contribute to resistance of EVI $1^{\text {high }}$ AML to RA agonists such as ATRA.

In our study of the exact $\mathrm{t}(3 ; 21)(\mathrm{q} 26 ; \mathrm{q} 11)$ breakpoints, as reported by Haferlach et $a .^{8}$ and D'Angiò et $a l .{ }^{9}$ we discovered that instead of forming a novel fusion protein, this translocation results in a repositioning of the complete EVII open reading frame under the control of the NRIP1 gene promoters and upstream regulatory elements containing multiple RA responsive elements. In chromosome 21 intact cells, these elements mediate a strong induction of NRIP1 transcription upon stimulation with ATRA. Treating $\mathrm{t}(3 ; 21)(q 26 ; q 11)$ cases with ATRA, as suggested in a recent clinical study, ${ }^{43}$ would therefore coordinate a similarly pronounced upregulation of the EVI1 oncogene in these special cases, presumably with devastating consequences. Another finding of our analyses of NRIP1 regulation is that the absence of RA receptor signaling - as in $\mathrm{t}(15 ; 17) \mathrm{AML}$ - abrogates NRIP1 expression. Transferring this knowledge to the aberrant NRIP1-abstracted upstream control of EVI1 that is unique to $t(3 ; 21)(q 26 ; q 11) A M L$ would therefore theoretically open a therapeutic window for RA receptor antagonists, which might help to reduce or even abrogate expression of the EVI1 oncogene in these cases. Our data and those from Nguyen et al. as well as a recent clinical study do not convincingly show a benefit for adding ATRA to the treatment of EVI1-expressing AML patients. ${ }^{43}$ As knockdown of NRIP1 negatively affected the proliferation and survival of EVI1-expressing AML cells, our findings warrant further investigation of NRIP1 as a therapeutic target in myeloid diseases with EVI1 activation.

\section{Disclosures}

No conflicts of interest to disclose.

\section{Contributions}

SG performed experiments, conducted data analyses and wrote the manuscript. $A C$ and $L M$ established and performed the knockdown experiments and NRIP1 RNA and protein quantification. Jl performed flow cytometry and western blots, and quantified relative band intensities. $C R, B D$, and NS helped to perform the CRISPR experiments and quantitative reverse transcriptase polymerase chain reaction measurements. $F R, K D$ and $L B$ provided clinical samples, clinical data, and input to the manuscript. JRP contributed the funding and access to facilities for RNA sequencing to generate the CK-AML dataset and revised the manuscript. TH gave advice on the study design and data analyses and revised the manuscript. AR and FK supervised the study and revised the manuscript.

\section{Acknowledgments}

The authors would like to thank Dr. Dirk Heckl for providing 
the pL4OC-CRISPR.EFS.PAC and $p L-C R I S P R$.EFS.tRFP vectors and Dr. Jan Krönke for providing the Cas9-expressing OClAML-5 cell line.

\section{Funding}

SG is supported by the Deutsche Forschungsgemeinschaft (DFG, German Research Foundation - project 446251518), the Michael Smith Foundation for Health Research (MSFHR) and the Lotte \& John Hecht Memorial Foundation (project RT2020-0578). AR was supported by the DFG (SFB 1074, project $A 5$, and the gender equality program SFB 1074, project Z2). FK was supported by the DFG (SFB 1074, project A5), the BC Cancer Foundation and the Leukemia \& Lymphoma Society of Canada.

\section{Data-sharing statement}

All datasets that were analyzed for the current study are available in the NCBI's GEO repository under the indicated identifiers, which are listed in the Methods section and cited throughout the main text and figure legends. RNA-sequencing data from the CK-AML cohort will be made available upon request. Please contact the corresponding author.

\section{References}

1. Gröschel S, Lugthart S, Schlenk RF, et al. High EVI1 expression predicts outcome in younger adult patients with acute myeloid leukemia and is associated with distinct cytogenetic abnormalities. J Clin Oncol. 2010;28(12):2101-2107.

2. Ayoub E, Wilson MP, McGrath KE, et al. EVI1 overexpression reprograms hematopoiesis via upregulation of Spi1 transcription. Nat Commun. 2018;9(1):4239.

3. Stein, S, Ott M, Schultze-Strasser S, et al. Genomic instability and myelodysplasia with monosomy 7 consequent to EVI1 activation after gene therapy for chronic granulomatous disease. Nat Med. 2010;16(2):198-204.

4. van Doorn SBW, Erpelinck CAJ, van Putten WLJ, et al. High EVI1 expression predicts poor survival in acute myeloid leukemia: a study of 319 de novo AML patients. Blood. 2003;101(3):837-845.

5. Lugthart S, van Drunen E, van Norden Y, et al, High EVI1 levels predict adverse outcome in acute myeloid leukemia: prevalence of EVI1 overexpression and chromosome 3q26 abnormalities underestimated. Blood. 2008;111(8):4329-4337.

6. Rockova V, Abbas S, Wouters BJ, et al. Risk stratification of intermediate-risk acute myeloid leukemia: integrative analysis of a multitude of gene mutation and gene expression markers. Blood. 2011;118(4):1069-1076.

7. Haas K, Kundi M, Sperr WR, et al. Expression and prognostic significance of different mRNA 5 '-end variants of the oncogene EVI1 in 266 patients with de novo AML: EVI1 and MDS1/EVI1 overexpression both predict short remission duration. Genes Chromosomes Cancer. 2008;47(4):288-298.

8. Haferlach C, Bacher U, Grossmann, et al. Three novel cytogenetically cryptic EVI1 rearrangements associated with increased EVI1 expression and poor prognosis identified in 27 acute myeloid leukemia cases. Genes Chromosomes Cancer. 2012;51(12):1079-1085.

9. D'Angiò M, Fazio G, Grioni A, et al. High EVI1 expression due to NRIP1/EVI1 fusion in therapy-related acute myeloid leukemia: description of the first pediatric case. Hemasphere. 2020;17;4(5):e471.

10. Pombo A, Dillon N. Three-dimensional genome architecture: players and mechanisms. Nat Rev Mol Cell Biol. 2015;16(4):245-257.

11. L'Horset F, Dauvois S, Heery DM, et al. RIP-140 interacts with multiple nuclear receptors by means of two distinct sites. Mol Cell Biol. 1996;16(11):6029-6036.

12. Vivante $\mathrm{A}$, Mann $\mathrm{N}$, Yonath $\mathrm{H}$, et al. A dominant mutation in nuclear receptor interacting protein 1 causes urinary tract malformations via dysregulation of retinoic acid signaling. J Am Soc Nephrol. 2017;28(8):2364-2376.

13. Cabezas-Wallscheid N, Buettner F, Sommerkamp P et al.
Vitamin A-retinoic acid signaling regulates hematopoietic stem cell dormancy. Cell. 2017;169(5):807-823.

14. Augereau P, Badia E, Carascossa S, et al. The nuclear receptor transcriptional coregulator RIP140. Nucl Recept Signal. 2006; 4:e024.

15. The Cancer Genome Atlas Research Network. Genomic and epigenomic landscapes of adult de novo acute myeloid leukemia. N Engl J Med. 2013;368(22):2059-2074.

16. Tyner JW, Tognon CE, Bottomly D, et al. Functional genomic landscape of acute myeloid leukaemia. Nature. 2018;562(7728):526-531.

17. Martens JHA, Stunnenberg HG. BLUEPRINT: mapping human blood cell epigenomes. Haematologica. 2013;98(10):1487-1489.

18. Pellagatti A, Armstrong RN, Steeples V, et al. Impact of spliceosome mutations on RNA splicing in myelodysplasia: dysregulated genes/pathways and clinical associations. Blood. 2018;132(12):1225-1240.

19. Herold T, Jurinovic V, Batcha AMN, et al. A 29-gene and cytogenetic score for the prediction of resistance to induction treatment in acute myeloid leukemia. Haematologica. 2018;103(3):456-465.

20. Hirsch S, Blätte TJ, Grasedieck S, et al. Circular RNAs of the nucleophosmin (NPM1) gene in acute myeloid leukemia. Haematologica. 2017;102(12):2039-2047.

21. Saito Y, Nakahata S, Yamakawa, et al. CD52 as a molecular target for immunotherapy to treat acute myeloid leukemia with high EVI1 expression. Leukemia. 2011;25(6):921-931.

22. Nguyen $\mathrm{CH}$, Bauer $\mathrm{K}$, Hackl $\mathrm{H}$, et al. All-trans retinoic acid enhances, and a pan-RAR antagonist counteracts, the stem cell promoting activity of EVI1 in acute myeloid leukemia. Cell Death Dis. 2019;10(12):944.

23. ENCODE Project Consortium. An integrated encyclopedia of DNA elements in the human genome. Nature. 2012;489(7414):57-74.

24. Gertz J, Savic D, Varley KE, et al. Distinct properties of celltype-specific and shared transcription factor binding sites. Mol Cell. 2013;52(1):25-36.

25. Rousseau M, Ferraiuolo MA, Crutchley JL, et al. Classifying leukemia types with chromatin conformation data. Genome Biol. 2014;15(4):R60.

26. Loke J, Assi SA, Imperato MR, et al. RUNX1-ETO and RUNX1-EVI1 differentially reprogram the chromatin landscape in $\mathrm{t}(8 ; 21)$ and t(3;21) AML. Cell Rep. 2017;19(8):1654-1668.

27. Tang Z, Luo OJ, Li X, et al. CTCF-mediated human 3D genome architecture reveals chromatin topology for transcription. Cell. 2015;163(7):1611-1627.

28. Rao S, Huntley MH, Durand NC, et al. A 3D map of the human 
genome at kilobase resolution reveals principles of chromatin looping. Cell. 2014;159(7):1665-1680.

29. Phanstiel DH, Van Bortle K, Spacek D, et al. Static and dynamic DNA loops form AP-1-bound activation hubs during macrophage development. Mol Cell. 2017;67(6):1037-1048.

30. Dixon JR, Jung I, Selvaraj S, et al. Chromatin architecture reorganization during stem cell differentiation. Nature. 2015;518(7539):331-336.

31. Rubin A, Barajas B, Furlan-Magaril M, et al. Lineage-specific dynamic and pre-established enhancer-promoter contacts cooperate in terminal differentiation. Nat Genet. 2017;49(10):1522-1528.

32. Kassambara A, Kosinski M. (2018). survminer: drawing survival curves using 'ggplot2'. R package version 0.4.2. https://CRAN.Rproject.org/package=survminer.

33. Therneau T (2015). A package for survival analysis in S. version 2.38,. https://CRAN.R-project.org/package=survival.

34. Hothorn T, Lausen B. On the exact distribution of maximally selected rank statistics. Comput Stat Data Anal. 2002;43(2):121-137.

35. Gröschel S, Sanders MA, Hoogenboezem R, et al. A single oncogenic enhancer rearrangement causes concomitant EVI1 and GATA2 deregulation in leukemia. Cell. 2014;157(2):369-381.

36. Yamazaki H, Suzuki M, Otsuki A, et al. A remote GATA2 hematopoietic enhancer drives leukemogenesis in inv(3)(q21;q26) by activating EVI1 expression. Cancer Cell. 2014;25(4):415-427.

37. Rücker FG, Gong X, Dolnik A, et al. Identification of novel gene fusions in acute myeloid leukemia with complex karyotype by transcriptome analysis using RNA sequencing. Haematologica. 2017;102(s2):39-40.

38. Fishilevich S, Nudel R, Rappaport N, et al. GeneHancer: genomewide integration of enhancers and target genes in GeneCards. Database (Oxford). 2017;2017:bax028.

39. Kerley JS, Olsen SL, Freemantle SJ, et al. Transcriptional activation of the nuclear receptor corepressor RIP140 by retinoic acid: a potential negative-feedback regulatory mechanism. Biochem Biophys Res Commun. 2001;285(4):969-975.

40. Tohda S, Kurokawa H, Nara N. Relation of protein kinase A and protein kinase $C$ to signaling pathways of hematopoietic factors in leukemia cell lines. Int J Oncol. 1996;8(3):521-524.

41. Huret JL. t(3;21)(q26;q22). Atlas Cytogenet Oncol Haematol. 2014;18(1):53-56. Online version: http://AtlasGeneticsOncology.org/Anomalies/t0321ID1009.html.

42. Schoenfelder S, Fraser P. Long-range enhancer-promoter contacts in gene expression control. Nat Rev Genet. 2019;20(8):437-455

43. Paubelle E, Plesa A, Hayette S, et al. Efficacy of all-transretinoic acid in high-risk acute myeloid leukemia with overexpression of EVI1. Oncol Ther. 2019;7(2):121-130. 